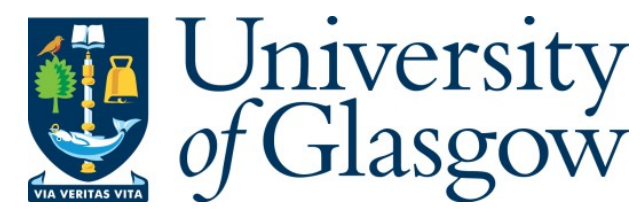

Heiligers, J., M clnnes, C.R ., B iggs, J.D., and Ceriotti, M . (2012) Displaced geostationary orbits using hybrid low-thrust propulsion.A cta A stronautica, 71. pp. 51-67. ISSN 0094-5765

Copyright $\odot 2012$ Elsevier

A copy can be downloaded for personal non-commercial research or study, without prior permission or charge

Content must not be changed in any way or reproduced in any format or medium without the formal permission of the copyright holder(s)

When referring to this work, full bibliographic details must be given

$\underline{\text { http://eprints.gla.ac.uk/68858/ }}$

Deposited on: 11 February 2014

Enlighten - Research publications by members of the University of Glasgow http://eprints.gla.ac.uk 


\title{
Displaced Geostationary Orbits Using Hybrid Low-Thrust Propulsion*
}

\author{
Jeannette Heiligers ${ }^{a, \dagger}$, Colin R. McInnes ${ }^{\text {a»}, ~ J a m e s ~ D . ~ B i g g s ~}{ }^{\text {å }}$, Matteo Ceriotti ${ }^{a * *}$ \\ ${ }^{a}$ Advanced Space Concepts Laboratory, Department of Mechanical Engineering, University of \\ Strathclyde, James Weir Building, 75 Montrose Street, G1 1XJ Glasgow, United Kingdom
}

In this paper, displaced geostationary orbits using hybrid low-thrust propulsion, a complementary combination of Solar Electric Propulsion (SEP) and solar sailing, are investigated to increase the capacity of the geostationary ring that is starting to become congested. The SEP propellant consumption is minimized in order to maximize the mission lifetime by deriving semi-analytical formulae for the optimal steering laws for the SEP and solar sail accelerations. By considering the spacecraft mass budget, the performance is also expressed in terms of payload mass capacity. The analyses are performed both for the use of pure SEP and hybrid low-thrust propulsion to allow for a comparison. It is found that hybrid lowthrust control outperforms the pure SEP case both in terms of payload mass capacity and mission lifetime for all displacements considered. Hybrid low-thrust propulsion enables payloads of 255 to $487 \mathrm{~kg}$ to be maintained in a $35 \mathrm{~km}$ displaced orbit for 10 to 15 years. Adding the influence of the $J_{2}$ and $J_{2,2}$ terms of the Earth's gravity field has a small effect on this lifetime, which becomes almost negligible for small values of the sail lightness number. Finally, two SEP transfers that allow for an improvement in the performance of hybrid low-thrust control are optimized for the propellant consumption by solving the accompanying optimal control problem using a direct pseudospectral method. The first type of transfer enables a transit between orbits displaced above and below the equatorial plane, while the second type of transfer enables customized service for which a spacecraft is transferred to a Keplerian parking orbit when geostationary coverage is not needed. While the latter requires a modest propellant budget, the first type of transfer comes at the cost of an almost negligible SEP propellant consumption.

Keywords: Displaced non-Keplerian orbits, Geostationary orbit, Solar sailing, Hybrid low-thrust propulsion, Trajectory optimization

\section{Introduction}

Since the first geostationary spacecraft was launched in 1964, Syncom-3, hundreds of communication and weather satellites have exploited the unique properties of the geostationary orbit (GEO). With a period equal to the Earth's rotational period, GEO spacecraft are stationary with respect to their ground station, allowing for a continuous downlink to Earth. However, with only one such unique orbit, the geostationary orbit has started to become congested over time. Ref. [1] reports the status of the geostationary orbit in terms of controlled and uncontrolled spacecraft in January 2009 and clearly shows its congestion, especially above the continents. The situation becomes even worse when considering the fact that, besides

\footnotetext{
* This paper was presented during the $61^{\text {st }}$ International Astronautical Congress in Prague, Czech Republic

${ }^{\dagger}$ Corresponding author. Tel.:+44 141548 5989. E-mail address: Jeannette.Heiligers@strath.ac.uk

Tel.:+44 141548 2049. E-mail address: Colin.McInnes@strath.ac.uk

§ Tel.:+44 141548 2042. E-mail address: James.Biggs@strath.ac.uk

** Tel.:+44 141548 5726. E-mail address: Matteo.Ceriotti@strath.ac.uk
} 
these (un)controlled spacecraft, the geostationary orbit also contains fragmentation debris, rocket bodies and mission-related objects.

In order to increase the capacity of the geostationary orbit, this paper investigates the use of displaced non-Keplerian orbits (NKOs). By applying a continuous acceleration to counterbalance the gravitational acceleration, the geostationary orbit can be levitated above or below the equatorial plane, thereby creating new geostationary slots [2]. The existence, stability and control of displaced NKOs have been studied for both the two- and three-body problem [3-4] and numerous applications have been proposed. The two-body problem applications include spacecraft proximity operations [5] and hovering above Saturn's rings for insitu observations [6]. NKOs displaced high above the ecliptic have been proposed in the Earth-Sun threebody problem to enable imaging and communication satellites for high latitudes [7], while displaced NKOs in the Earth-Moon system have been studied for lunar far side communication and lunar south pole coverage [8-9].

Solar sails have often been proposed as spacecraft propulsion system to maintain displaced NKOs [2, 4, 7 8, 10]. Solar sails exploit the radiation pressure generated by photons reflecting off a large, highly reflecting sail to produce a continuous, propellant-less thrust [2]. This makes them seemingly suitable to maintain displaced NKOs. However, only recently solar sail technology was successfully demonstrated in space by the Japanese IKAROS spacecraft [11] and NASA's NanoSail-D mission [12]. Despite these advances, the Technology Readiness Level (TRL) of solar sailing as primary propulsion system on a reasonable sized mission is still rather low. That, in combination with a high Advanced Degree of Difficulty $\left(\mathrm{AD}^{2}\right)$ and the inability to generate a thrust component in the direction of the Sun, poses severe limits on its applications and puts many solar sail applications in the far-future [2,13]. Solar sails have also been proposed to make levitated geostationary orbits possible [14]. However only small displacements, still inside the geostationary station keeping box for near-future solar sails, appeared to be feasible and a residual in-plane sail acceleration caused the spacecraft to move with respect to its ground station.

Solar Electric Propulsion (SEP) has also been considered as a means to maintain displaced NKOs [15]. SEP is highly efficient as it enables high specific impulses. It has flown on multiple missions including the NSTAR ion engines on Deep Space 1 (1998) and Dawn (2007), the PPS1350 Hall thruster on SMART-1 (2003), the QinetiQ's T5 thrusters on GOCE (2009) and the Aerojet BPT4000 thruster on the Advanced Extremely High Frequency geostationary satellite (2010) [16-18]. This results in a high TRL and a low $\mathrm{AD}^{2}$. Nevertheless, the applications of SEP are limited due to a bound on the available propellant mass.

Considering the advantages and disadvantages of solar sails and SEP, some authors are suggesting the use of hybrid low-thrust propulsion, a complementing combination of a solar sail and an SEP system. While the solar sail lowers the demand on the SEP propellant mass, the SEP system can provide the thrust component in the direction of the Sun that the solar sail cannot generate and lower the solar sail $\mathrm{AD}^{2}$ as only small solar sails are required. Hybrid low-thrust propulsion has been suggested to enable interplanetary transfers [19-20], to allow for periodic orbits in the vicinity of the Lagrange points in the Earth-Moon system for lunar communication purposes [21], and to generate artificial equilibria in the Earth-Sun three-body problem [22], for instance for an Earth-Mars communications relay during periods of solar occultation [23] and to enable an Earth pole-sitter [24]. All studies show to some extent an improvement for hybrid low-thrust propulsion over the use of pure SEP or pure solar sailing in terms of propellant mass consumption, required thrust magnitude levels and/or initial spacecraft mass.

Building upon these findings, we propose to use hybrid low-thrust propulsion to enable displaced geostationary orbits. This will allow spacecraft to be stationary with respect to their ground station and enable displacements well beyond the geostationary station keeping box, using relatively small, near-term solar sails. These solar sails could possibly also provide a solution to comply with the Inter-Agency Space Debris Coordination Committee (IADC) mitigation guidelines that request that spacecraft are removed 
from densely populated orbital regions once they have reached their end of life, and as such prevent further congestion of the geostationary zone. The objective is to minimize the propellant consumption, thereby either decreasing launch mass, increasing payload mass or increasing the mission lifetime. To assess the performance of hybrid low-thrust control, its results are compared with results for the use of pure SEP control and the influence of the $J_{2}$ and $J_{2,2}$ terms of the Earth's gravity field is investigated. Finally, the optimization of two transfers that improve the performance of hybrid low-thrust control will be considered: a transfer between orbits displaced above and below the equatorial plane and a transfer between the displaced orbit and a Keplerian parking orbit to enable customized service.

The structure of the paper is as follows. First the general theory underlying displaced geostationary orbits will be presented. Subsequently the performance of SEP and hybrid low-thrust control in terms of propellant consumption will be derived and a comparison between the two control strategies will be made. Then, the influence of the non-uniform Earth's gravity field on the propellant consumption will be investigated. A mass budget analysis will subsequently consider the performance of both types of control in terms of payload mass capacity. Finally, the analysis to optimize the two transfers that improve the performance of hybrid low-thrust control will be outlined and the results will be presented.

\section{Displaced geostationary orbits}

Displaced geostationary orbits, or displaced NKOs in general, can be found by seeking equilibrium solutions to the two-body problem in a rotating frame of reference. A transformation to an inertial frame will subsequently show that the spacecraft executes a circular orbit displaced away from the center of the central body [3]. The situation as it occurs in the displaced geostationary orbit is depicted in Fig. 1, indicating the rotating reference frame $R\left(x_{R}, y_{R}, z_{R}\right)$ that rotates with constant angular velocity $\omega=\omega \hat{\mathbf{z}}_{R}$ with respect to an inertial frame $I(X, Y, Z)$. The figure shows that the geostationary orbit is levitated over a distance $h$ while keeping both the orbital radius and the orbital angular velocity equal to the orbital radius and orbital angular velocity in the geostationary orbit, $r_{G E O}$ and $\omega$ respectively, causing spacecraft in the (displaced) geostationary orbit to be stationary in the rotating frame. This case corresponds to a 'Type I' NKO for which the thrust induced acceleration, a, required to maintain the NKO is at its minimum for a given radius of the NKO and which is stable for modest displacements [2]. Following the analysis in Ref. [3], the required direction (i.e, the pitch angle $\alpha$ (see Fig. 1)) and magnitude of this acceleration are:

$$
\begin{aligned}
\tan \alpha & =0 \\
a & =h \omega^{2}=\frac{\mu h}{r_{G E O}^{3}}
\end{aligned}
$$

with $\mu$ the gravitational parameter of the Earth. Eq. (1) shows that a thrust perpendicular to the displaced geostationary orbit is required and that the magnitude of the thrust is merely a function of the gravitational parameter, the displacement distance and the orbital radius. Note that for a geostationary orbit displaced above the equatorial plane $(h>0)$ the required acceleration is directed in positive $Z$ direction, while for orbits displaced below the equatorial plane $(h<0)$ the acceleration is directed in negative $Z$ direction.

With the gravitational parameter and the orbital radius given, the only parameter that needs to be specified is the displacement distance, which is derived from the height of the geostationary station keeping box in order to prevent interference and collisions with spacecraft in the geostationary orbit. In terms of longitude, station keeping regulations currently require a station keeping box height of $\Delta \lambda=0.05^{\circ} \sim 0.1^{\circ}$, which equals $36.8 \sim 73.6 \mathrm{~km}$ [25-26]. Since the displaced geostationary spacecraft will be an actively controlled satellite, one could argue that the displacement distance would only have to be $\Delta \lambda$ such that the 
spacecraft hovers exactly on top of the station keeping box. However, to ensure a similar station keeping box for the displaced spacecraft as for geostationary spacecraft, the displacement distance might have to be increased to $2 \Delta \lambda$, leading to a range for the displacement distance of $36.8 \sim 147.2 \mathrm{~km}$. This paper will therefore consider three different displacement distances, namely 35, 75 and $150 \mathrm{~km}$ both above and below the equatorial plane.

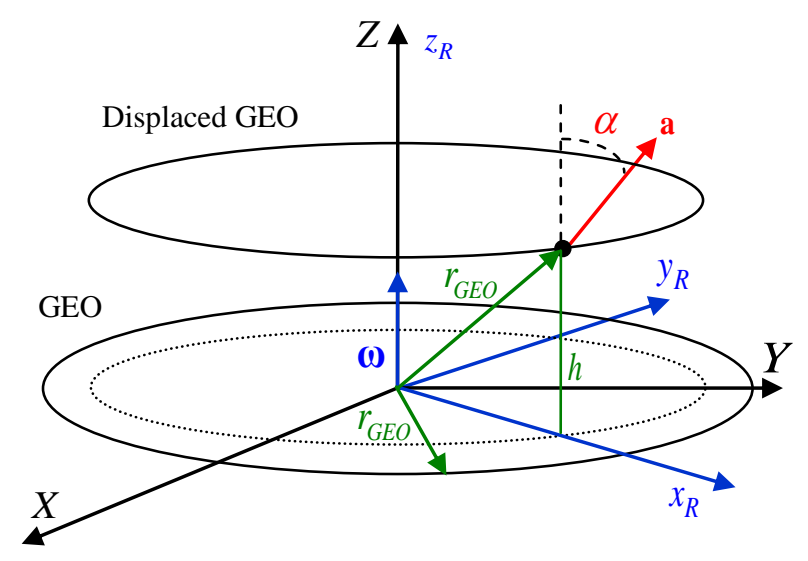

Fig. 1: Definition of displaced geostationary orbit.

\section{Solar electric propulsion}

This section investigates the use of SEP to provide the continuous acceleration required to maintain the displaced geostationary orbit, see Eq. (1). Its performance in terms of propellant consumption can be assessed by integrating the following differential equation for the mass:

$$
\dot{m}=-\frac{T}{I_{s p} g_{0}}
$$

with $T$ the SEP thrust magnitude, $I_{s p}$ the SEP system specific impulse and $g_{0}$ the Earth gravity constant $\left(9.80665 \mathrm{~m} / \mathrm{s}^{2}\right.$ at the surface of the Earth). The lifetime of the mission, $L$, is subsequently defined as the epoch at which a particular mass fraction $m_{f} / m_{0}=\left(m_{0}-m_{\text {prop }}\right) / m_{0}$ is obtained, with $m_{0}$ the initial mass, $m_{f}$ the final mass (i.e. the mass at lifetime $L$ ) and $m_{\text {prop }}$ the propellant mass. The lifetime can be derived analytically from Eq. (2) as the required acceleration is constant. Substituting $T=a \cdot m$ into Eq. (2) with $a$ given by Eq. (1) and rearranging gives:

$$
\int_{m_{0}}^{m_{f}} \frac{d m}{m}=-\int_{t_{0}}^{t_{f}} \frac{a}{I_{s p} g_{0}} d t
$$

Evaluating these integrals and setting $t_{0}=0$ yields the following lifetime for a particular mass fraction:

$$
L=t_{f}=\ln \left(\frac{m_{0}}{m_{f}}\right) \frac{I_{s p} g_{0}}{a}
$$

Fig. 2 shows the lifetimes for the three displacement distances determined in Section 2 and for a wide range of mass fractions and specific impulses (from current to near term and far-future technology). Note 
that an arbitrary value for the initial mass can be assumed and that, due to the symmetry of the problem, the results for orbits displaced above and below the equatorial plane are exactly the same. Finally, only lifetimes up to 15 years are considered. Fig. 2 shows that, for example, for a $35 \mathrm{~km}$ displaced geostationary orbit, a currently feasible specific impulse of 3200 s (e.g. as flown on the Hayabusa spacecraft [27]) and a mass fraction of 0.5 , a lifetime of 3.7 years can be achieved. However, this lifetime degrades to 1.7 and 0.9 years when considering the larger displacements of 75 and $150 \mathrm{~km}$, respectively.

For current geostationary spacecraft, the lifetime is limited to $10 \sim 15$ years, mainly due to the required costly station keeping. Fig. 2 shows that similar lifetimes can only be achieved for the smallest displacement of $35 \mathrm{~km}$ and either for low mass fractions (e.g. $m_{f} / m_{0}=0.1$ and $I_{s p}=2600 \mathrm{~s}$ ) or for farfuture specific impulses (e.g. $m_{f} / m_{0}=0.45$ and $I_{s p}=7500 \mathrm{~s}$ ).
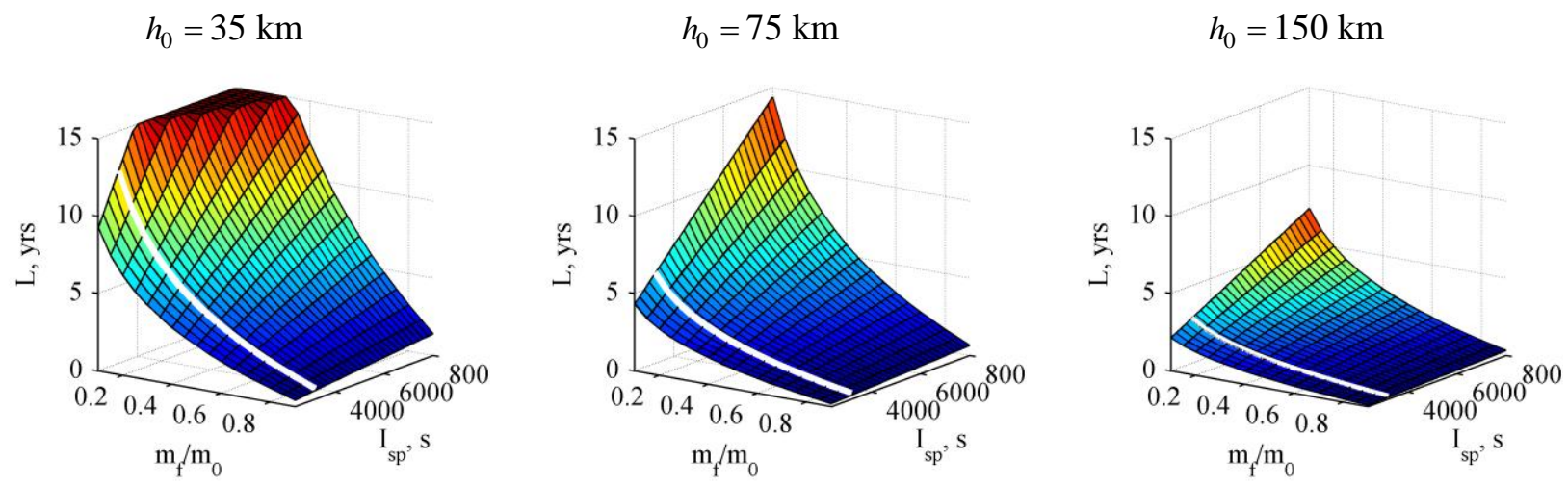

Fig. 2: Displaced geostationary orbits maintained with SEP control: mission time $L$ (maximum of 15 years) as a function of the specific impulse $I_{s p}$ (where the line indicates a currently feasible specific impulse of 3200 s) and the mass fraction $m_{f} / m_{0}$, for different values of the displacement distance $h$.

\section{Hybrid low-thrust propulsion}

To improve the performance of SEP control in terms of propellant consumption, this section will investigate the use of hybrid low-thrust propulsion to maintain the displaced geostationary orbit. To assess its performance, the equations of motion for a spacecraft in the displaced geostationary orbit are considered using the rotating reference frame given in Fig. 1:

$\ddot{\mathbf{r}}+2 \boldsymbol{\omega} \times \dot{\mathbf{r}}+\nabla U=\mathbf{a}$

with $\mathbf{r}$ the position vector, $\mathbf{r}=\left[\begin{array}{lll}x_{R} & y_{R} & z_{R}\end{array}\right]^{T}, U$ a potential that combines the gravitational potential of the central body and a potential representing the centripetal acceleration. The acceleration required to maintain the displaced geostationary orbit can be written as the sum of the acceleration generated by the SEP system, $\mathbf{a}_{S E P}$, and the acceleration produced by the solar sail, $\mathbf{a}_{s}$ :

$\mathbf{a}=\mathbf{a}_{S E P}+\mathbf{a}_{s}$

To maximize the lifetime of the spacecraft, the objective is to minimize the propellant consumption, or equivalently to minimize the magnitude of the acceleration required from the SEP system:

$\min \left(a_{S E P}\right)=\min \left(\left\|\mathbf{a}-\mathbf{a}_{s}\right\|\right)$ 
The required acceleration, a, is given through Eq. (1), while the acceleration generated by an ideal (i.e. a perfectly reflecting) solar sail is given by:

$\mathbf{a}_{s}=\beta \frac{\mu_{S}}{r_{s}^{2}}\left(\hat{\mathbf{n}} \cdot \hat{\mathbf{r}}_{S}\right)^{2} \hat{\mathbf{n}}$

$\mu_{S}$ is the gravitational parameter of the Sun, $\mathbf{r}_{s}$ is the Sun-sail vector and $\hat{\mathbf{n}}$ is the unit vector in the direction of the solar radiation pressure force. Note that for a perfectly reflecting solar sail as considered here, $\hat{\mathbf{n}}$ is directed normal to the sail surface. The magnitude of the Sun-sail vector is approximated by a constant Sun-Earth distance of $1 \mathrm{AU}$, leading to a maximum approximation error in $r_{s}$ of 1.7 percent. Finally, $\beta$ is the solar sail lightness number and can be defined as the ratio between the system loading, $\sigma$ (i.e. the ratio of the spacecraft mass to the solar sail area, $\sigma=m / A_{s}$ ) and the critical sail loading, $\sigma^{*}=1.53 \mathrm{~g} / \mathrm{m}^{2}[2]: \beta=\sigma^{*} / \sigma$. The sail lightness number is therefore a function of the spacecraft mass. Since the mass of the hybrid low-thrust spacecraft will decrease due to the consumption of propellant by the SEP system, the parameter $\beta$ increases according to $\beta=\beta_{0} m_{0} / m$, where the subscript ' 0 ' indicates the start of the mission at time $t=0$.

Due to the tilt of the Earth's rotational axis with respect to the ecliptic plane, the direction of the Sun-sail vector $\mathbf{r}_{s}$ changes during the year. To model this variation, an Earth fixed rotating reference frame $E\left(x_{E}, y_{E}, z_{E}\right)$ as shown in Fig. 3 is used. Centered at the Earth with the $\left(x_{E}, y_{E}\right)$ plane in the equatorial plane and the $z_{E}$ axis along the rotational axis of the Earth, this reference frame rotates with the same angular velocity as the orbit of the Earth, causing the unit vector $\hat{\mathbf{r}}_{s}$ to always be contained in the $\left(x_{E}, z_{E}\right)$ plane. The angle $\chi$ describes the time during the year (with $\chi=0$ at winter solstice), while the angle $\psi$ is defined as the angle between $\hat{\mathbf{r}}_{s}$ and the equatorial plane as a function of $\chi$. This angle is at its maximum at winter solstice $\left(\psi(0)=i_{o b l}\right)$ and at its minimum in summer solstice $\left(\psi(\pi)=-i_{o b l}\right)$ with $i_{o b l}$ the obliquity of the ecliptic. The magnitude of $\psi$ is therefore equal to the solar declination, but is opposite in sign:

$\psi(\chi)=\sin ^{-1}\left(\sin i_{o b l} \cos \chi\right)$

Using this definition for $\psi$ gives the orientation of the Sun-sail line as $\hat{\mathbf{r}}_{s}=\left[\begin{array}{lll}\cos \psi & 0 & \sin \psi\end{array}\right]^{T}$ in the reference frame $\left(x_{E}, y_{E}, z_{E}\right)$.

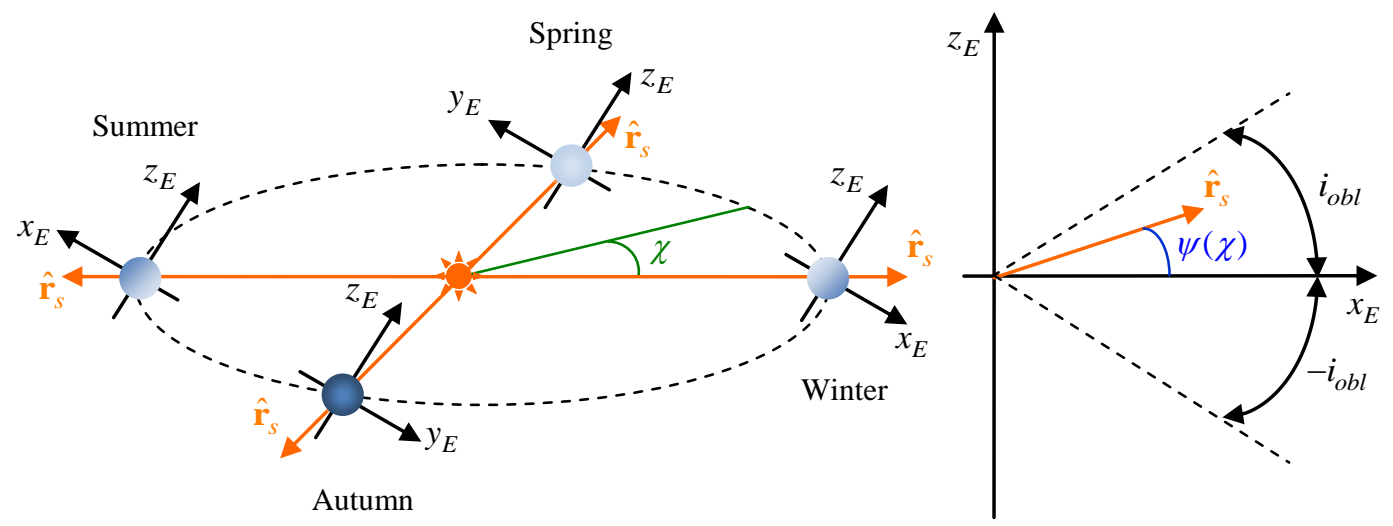

Fig. 3: Definition of reference frame and parameters used to model the seasonal variation of $\hat{\mathbf{r}}_{S}$. 


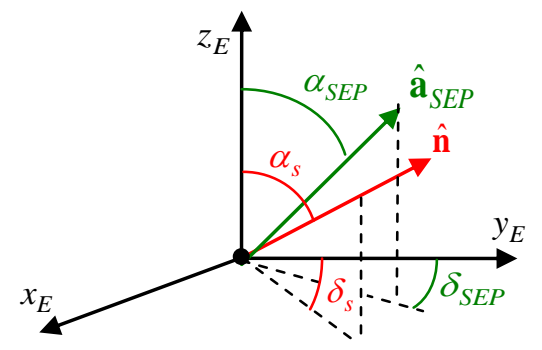

Fig. 4: Definition of solar sail and SEP pitch and yaw angles.

The unit vector normal to the sail surface, $\hat{\mathbf{n}}$, can be described using the same frame of reference, see Fig. 4. Using the pitch angle $\alpha_{s}$ and the yaw angle $\delta_{s}$, yields:

$\hat{\mathbf{n}}=\left(\begin{array}{c}\sin \alpha_{s} \sin \delta_{s} \\ \sin \alpha_{s} \cos \delta_{s} \\ \cos \alpha_{s}\end{array}\right)$

Substituting Eq. (1) and the expressions for $\hat{\mathbf{r}}_{s}, \hat{\mathbf{n}}$ and $\beta$ into Eq. (3) and rearranging gives:

$$
\begin{aligned}
& a_{S E P, x_{E}}=\quad-\beta_{0} \frac{m_{0}}{m} \frac{\mu_{S}}{r_{s}^{2}}\left(\cos \psi \sin \alpha_{s} \sin \delta_{s}+\sin \psi \cos \alpha_{s}\right)^{2} \sin \alpha_{s} \sin \delta_{s} \\
& a_{S E P, y_{E}}=\quad-\beta_{0} \frac{m_{0}}{m} \frac{\mu_{S}}{r_{s}^{2}}\left(\cos \psi \sin \alpha_{s} \sin \delta_{s}+\sin \psi \cos \alpha_{s}\right)^{2} \sin \alpha_{s} \cos \delta_{s} \\
& a_{S E P, z_{E}}=\frac{\mu h}{r_{G E O}^{3}}-\beta_{0} \frac{m_{0}}{m} \frac{\mu_{S}}{r_{s}^{2}}\left(\cos \psi \sin \alpha_{s} \sin \delta_{s}+\sin \psi \cos \alpha_{s}\right)^{2} \cos \alpha_{s}
\end{aligned}
$$

The SEP system thus needs to counterbalance the in-plane component of the solar sail acceleration and needs to augment the out-of-plane solar sail acceleration to obtain the required out-of-plane acceleration.

Inspecting Eq. (6) shows that for a given value for $m$ and $\psi$ (i.e. for a particular instant of time), the minimization problem in Eq. (4) is merely a function of the solar sail pitch and yaw angles and therefore reduces to finding the optimal solar sail pitch and yaw angles that minimize the acceleration required from the SEP system:

$$
\left(\alpha_{s}^{*}, \delta_{s}^{*}\right)=\underset{\substack{\alpha_{s} \in\left[\alpha_{s, \text { min }}, \alpha_{s, \text { max }}\right] \\ \delta_{s} \in[-\pi, \pi]}}{\arg \min }\left(a_{S E P}\left(\alpha_{s}, \delta_{s}\right)\right)
$$

where the domain of $\alpha_{s}$ is defined later in the paper. The solution to Eq. (7) can be found by setting the partial derivative of the SEP acceleration with respect to the sail pitch and yaw angles equal to zero:

$$
\frac{\partial a_{S E P}}{\partial \alpha_{s}}=\frac{\partial a_{S E P}}{\partial \delta_{s}}=0
$$

For this, the SEP acceleration is first written as:

$$
a_{S E P}=\sqrt{a_{S E P, x_{E}}^{2}+a_{S E P, y_{E}}^{2}+a_{S E P, z_{E}}^{2}}=\sqrt{c_{1}^{2}\left(\hat{\mathbf{n}} \cdot \hat{\mathbf{r}}_{S}\right)^{4}-2 c_{1} c_{2}\left(\hat{\mathbf{n}} \cdot \hat{\mathbf{r}}_{S}\right)^{2} \cos \alpha+c_{2}^{2}}
$$

with 
$c_{1}=\beta_{0} \frac{m_{0}}{m} \frac{\mu_{S}}{r_{s}^{2}}, \quad c_{2}=\frac{\mu h}{r_{G E O}^{3}}$

Taking the first derivative of Eq. (9) with respect to the yaw angle yields:

$$
\frac{\partial a_{S E P}}{\partial \delta_{s}}=\frac{1}{2 a_{S E P}} \frac{\partial}{\partial \delta_{s}}\left(c_{1}^{2}\left(\hat{\mathbf{n}} \cdot \hat{\mathbf{r}}_{s}\right)^{4}-2 c_{1} c_{2}\left(\hat{\mathbf{n}} \cdot \hat{\mathbf{r}}_{s}\right)^{2} \cos \alpha+c_{2}^{2}\right)=0
$$

from which the following condition can be derived:

$$
4 c_{1}\left(\hat{\mathbf{n}} \cdot \hat{\mathbf{r}}_{s}\right)\left(c_{1}\left(\hat{\mathbf{n}} \cdot \hat{\mathbf{r}}_{s}\right)^{2}-c_{2} \cos \alpha_{s}\right) \cos \psi \sin \alpha_{s} \cos \delta_{s}=0
$$

For Eq. (11) to hold throughout the year and considering that $c_{1} \neq 0$ and $\left(\hat{\mathbf{n}} \cdot \hat{\mathbf{r}}_{s}\right)>0$ (to generate a solar sail acceleration) the optimal yaw angle equals $\delta_{s}{ }^{*}= \pm \pi / 2$. Substituting this value into Eq. (6) shows that the $y_{E}$ component of the SEP thrust force is zero at all times. Considering the fact that the solar sail is unable to generate a thrust component in the direction of the Sun and recalling that the $x_{E}$ axis points away from the Sun at all times, the optimal yaw angle can be reduced to $\delta_{s}^{*}=\pi / 2$. This reduction also guarantees that $\partial^{2} a_{S E P} / \partial \delta_{s}^{2}>0$ such that the solution corresponds to a minimum rather than a maximum of $a_{S E P}\left(\alpha_{s}, \delta_{s}\right)$. A similar analysis can be performed for the partial derivative with respect to the sail pitch angle. Substituting $\delta_{s}=\delta_{s}^{*}=\pi / 2$ gives the condition:

$\sin \left(\alpha_{s}+\psi\right)-\frac{c_{2}}{c_{1}} \frac{\cos \alpha_{s}}{\sin \left(\alpha_{s}+\psi\right)}+\frac{c_{2}}{2 c_{1}} \frac{\sin \alpha_{s}}{\cos \left(\alpha_{s}+\psi\right)}=0$

An analytical solution for the optimal pitch angle was not found from this expression. A numerical method such as Newton's method (e.g. see Ref. [28]) is therefore applied to find $\alpha_{s}{ }^{*}$. To ensure that the optimal pitch angle does not generate a normal vector $\hat{\mathbf{n}}$ pointing towards the Sun, bounds are imposed on the optimum pitch angle. These bounds are a function of the angle $\psi$ as is shown in Fig. 5 for three epochs during the year. Then, to ensure $\partial^{2} a_{S E P} / \partial \alpha_{s}^{2}>0$, these bounds are set even tighter depending on whether a displacement above or below the equator is considered:

$$
\begin{aligned}
& \alpha_{s, \min }=\left\{\begin{array}{cc}
-\psi & h>0 \\
0.5 \pi & h<0
\end{array}\right. \\
& \alpha_{s, \max }=\left\{\begin{array}{cc}
0.5 \pi & h>0 \\
\pi-\psi & h<0
\end{array}\right.
\end{aligned}
$$

Note that Fig. 5 clearly illustrates that the displaced geostationary orbit as presented in this paper cannot be maintained throughout the year using only solar sailing. For instance, in summer the shaded half-circle shows that the required thrust direction for a geostationary orbit displaced above the equatorial plane (i.e. a thrust along the positive $z_{E}$ axis) cannot be achieved by the solar sail. A similar reasoning holds for a geostationary orbit displaced below the equatorial plane in winter. Furthermore, in autumn and spring the required thrust direction for orbits displaced both above and below the equator lies on the edge of the shaded half-circle. The magnitude of the solar sail acceleration along the $z_{E}$ axis in that case becomes equal to zero as the Sun shines edge-on to the solar sail. Then, a solar sail acceleration along the $z_{E}$ axis can only be achieved by tilting the normal vector $\hat{\mathbf{n}}$ away from the $z_{E}$ axis resulting in an acceleration 
component parallel to the equatorial plane, which has to be cancelled out by some other means such as an SEP system.

Winter

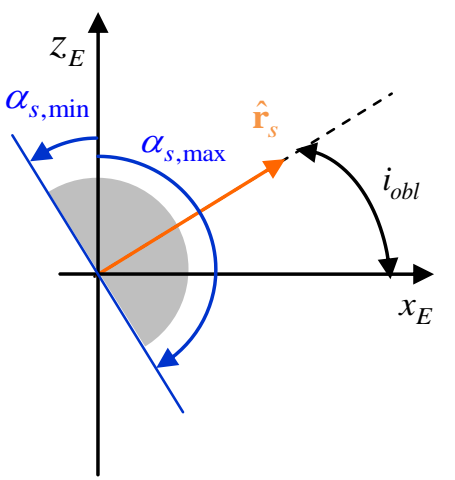

$\alpha_{s, \min }=-i_{o b l}, \alpha_{s, \max }=\pi-i_{o b l}$
Spring/Autumn

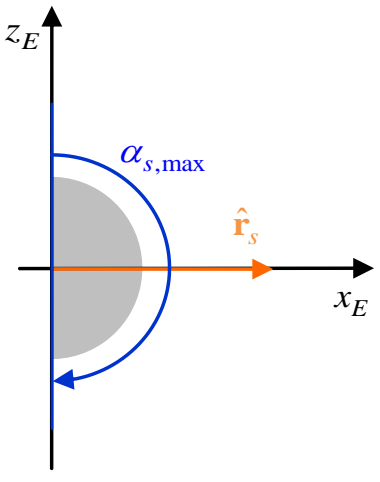

$\alpha_{s, \min }=0, \alpha_{s, \max }=\pi$
Summer

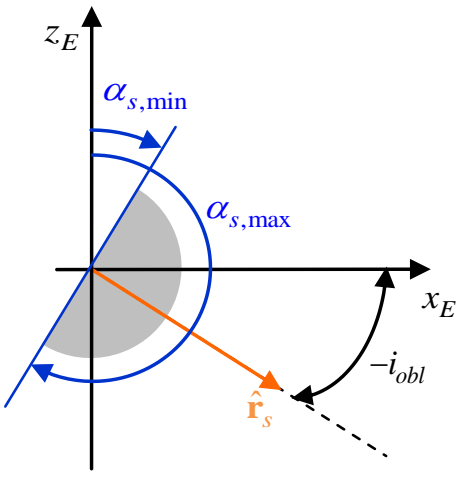

$\alpha_{s, \min }=i_{o b l}, \alpha_{s, \max }=\pi+i_{o b l}$

Fig. 5: Definition of minimum and maximum solar sail pitch angles during the year where the shaded half circle indicates the direction in which the solar sail can provide a thrust force.

Once the optimal sail pitch and yaw angles are found, the magnitude and direction of the required SEP acceleration can be computed. Note that the assumption is made that the solar sail and SEP system can steer independently of each other. Using Eq. (6) and the notation in Fig. 4, the pitch and yaw angles of the SEP acceleration can be computed:

$$
\begin{aligned}
& \alpha_{S E P}=\cos ^{-1}\left(\frac{a_{S E P, z_{E}}}{a_{S E P}}\right) \\
& \delta_{S E P}=\operatorname{atan} 2\left(a_{S E P, x_{E}}, a_{S E P, y_{E}}\right)
\end{aligned}
$$

as well as the magnitude of the required SEP thrust force:

$T=m \cdot a_{S E P}$

Previously it was already stated that $a_{S E P, y_{E}}=0$ since $\delta_{s}^{*}=\pi / 2$. Substituting this known into Eq. (13) gives $\delta_{S E P}= \pm \pi / 2$.

As mentioned before, the above holds for one instant in time, i.e. for a given value for $m$ and $\psi$. To find the variation of the controls, accelerations, thrust magnitude and mass as a function of time over multiple orbital periods, the displaced geostationary orbit is discretized into several nodes. When the node spacing is chosen small enough, a fair comparison with the analytical analysis in Section 3 can be made. The nodes are equally distributed over the orbit, leading to a constant time interval $\Delta t$ in between two consecutive nodes. At each node, $i$, the required SEP thrust magnitude can be approximated using Eq. (14) through $T_{i}=m_{i} \cdot a_{S E P, i}$. Assuming a constant thrust magnitude during the interval $\Delta t$, the mass at the end of the $i^{\text {th }}$ interval can be approximated through:

$m_{i+1}=m_{i}-\frac{T_{i}}{I_{s p} g_{0}} \Delta t$ 
At each node the optimum solar sail angles (and subsequently the SEP acceleration, thrust magnitude and thrust angles) can be computed. When changing from one node to the successive node, the change in $\psi$ is computed using Eq. (5), while the mass at the start of the new interval is given by Eq. (15).

The results after one year in a geostationary orbit displaced $35 \mathrm{~km}$ along the positive $z_{E}$ axis are shown in Fig. 6 and Fig. 7 (solid lines). A time interval of $\Delta t=0.005 t_{d a y}$ (with $t_{d a y}$ the length of a day) is adopted together with an initial mass of $1500 \mathrm{~kg}$ (the smaller class of geostationary spacecraft [29]) and a specific impulse of $3200 \mathrm{~s}$. Four different values for the sail lightness number are used, $\beta_{0}=0.01,0.05,0.1$ and 0.2. Some discontinuities can be observed in the profiles of the SEP thrust angles for the largest value of $\beta_{0}$, see Fig. 6a. This large value for $\beta_{0}$ causes the component of the solar sail acceleration along the positive $z_{E}$ axis to become larger than the required out-of-plane acceleration. This requires the SEP thruster to thrust along the negative $z_{E}$ axis to counterbalance the access out-of-plane acceleration, hence the switch in the SEP pitch angle from $\alpha_{S E P}<\pi / 2$ to $\alpha_{S E P}>\pi / 2$. Fig. 6a furthermore shows that the turn rate of the solar sail, which can often lead to operational difficulties, is very slow and has a maximum of approximately 40 degrees per half year for $\beta_{0}=0.2$. Another operational difficulty that may arise is the direction of the SEP thrust force with respect to the solar sail, which cannot be such that it lies in the plane of the solar sail. From the relative angle between the solar sail and SEP thrust forces, see Fig. 6b, it can be concluded that this is not the case for the displaced GEO.

Fig. 7a furthermore shows the expected lower demand on the SEP system by using hybrid low-thrust control, which is directly translated into a larger final mass after 1 year in-orbit. Already a solar sail with $\beta_{0}=0.01$ provides a gain of $29 \mathrm{~kg}$. Increasing $\beta_{0}$ results in savings of 94,130 and $161 \mathrm{~kg}$ for $\beta_{0}=0.05$, 0.1 and 0.2 , respectively.

a)

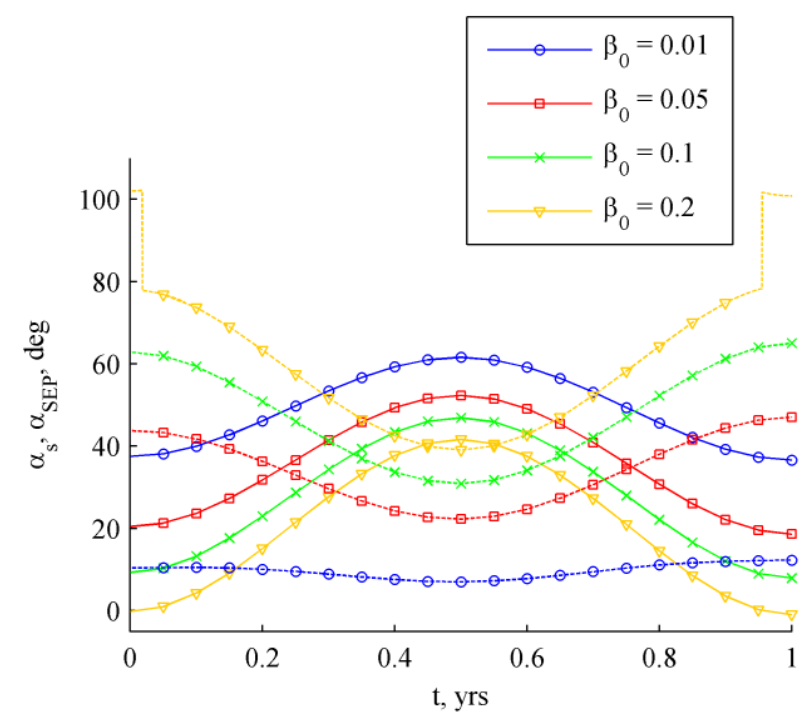

b)

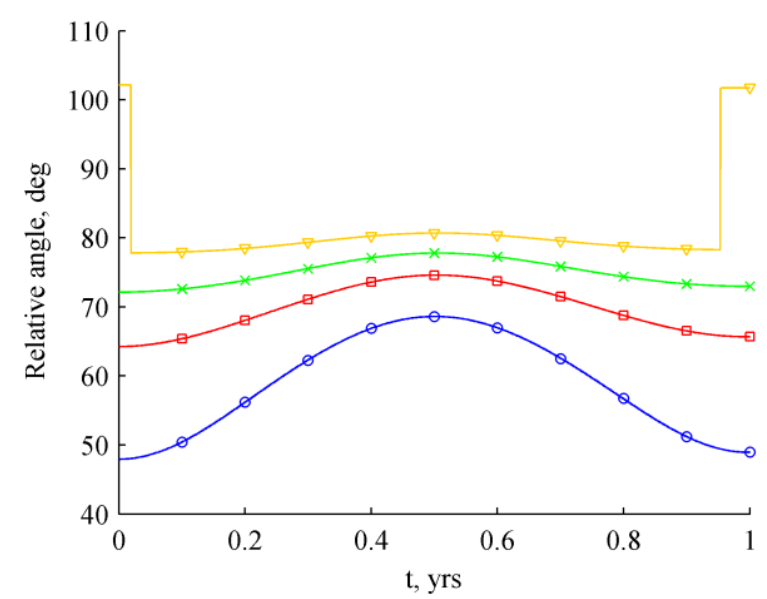

Fig. 6: Optimal solar sail (solid lines) and SEP (dashed lines) pitch angles (a) and relative angle between the solar sail and SEP thrust forces (b) for a geostationary orbit displaced $35 \mathrm{~km}$ above the equatorial plane maintained with hybrid low-thrust control with different values for the solar sail lightness number $\beta_{0}$. 
a)

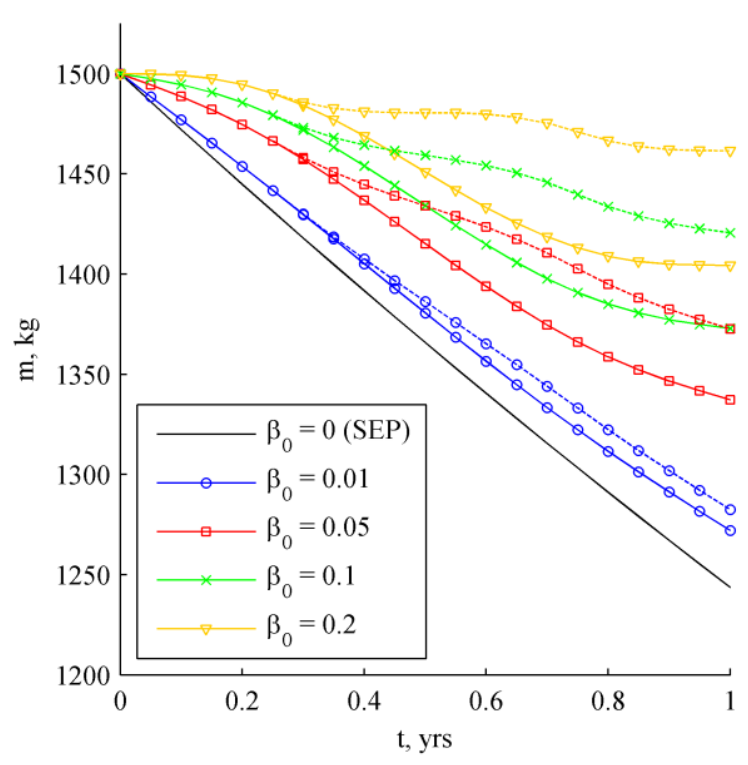

b)

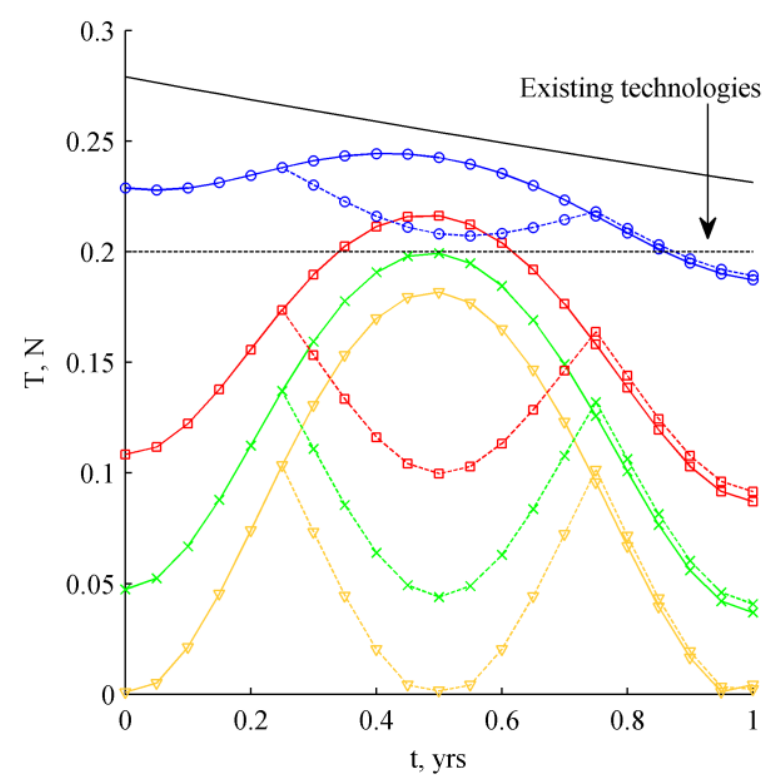

Fig. 7: $35 \mathrm{~km}$ displaced geostationary orbit maintained with hybrid low-thrust control for different values for the solar sail lightness number $\beta_{0}$. Spacecraft mass (a) and required SEP thrust magnitude (b) assuming an initial mass of $1500 \mathrm{~kg}$ and a specific impulse of $3200 \mathrm{~s}$. Solid lines indicate a year-long displacement along the positive $z_{E}$ axis. Dashed lines include a seasonal transfer between geostationary orbits displaced above and below the equatorial plane.

Finally, considering the required thrust magnitude in Fig. 7b, another great advantage of hybrid low-thrust propulsion over SEP becomes evident as hybrid low-thrust propulsion lowers the required SEP thrust magnitude. Currently feasible maximum thrust levels are in the order of $0.2 \mathrm{~N}$ at maximum power (e.g. EADS/Astrium RIT-XT). Note that larger thrust levels can be achieved by clustering multiple SEP thrusters. However, since this requires a larger initial mass (and with that launch mass and launch cost), an increased complexity of the spacecraft and an increase in the amount of power required, the current research assumes the use of one SEP thruster. Note that this also implies the assumption that one single thruster can meet the required propellant throughput and operational lifetime.

Fig. $7 \mathrm{~b}$ shows that, while the thrust level required for a $1500 \mathrm{~kg}$ spacecraft with SEP control exceeds the value of $0.2 \mathrm{~N}$, thrust levels smaller than $0.2 \mathrm{~N}$ throughout the year can be observed for $\beta_{0}=0.1$ and 0.2. Even for $\beta_{0}=0.05$ the thrust level remains well under $0.2 \mathrm{~N}$ during winter, but is too high during summer. This performance can be improved by transferring the spacecraft from a geostationary orbit displaced above the equatorial plane to an orbit displaced below the equatorial plane before summer. Then, the performance of the sail is no longer limited by the unfavorable obliquity of the ecliptic and can perform equally well in summer as it does in winter above the equatorial plane. When this so-called seasonal transfer is introduced in the model, results as presented by the dashed lines in Fig. 7 are obtained. Note that the mission is assumed to always start in winter, i.e. above the equatorial plane. As expected, improvements both in terms of propellant consumption and required thrust levels can be observed. The mass savings mentioned before are now increased to $39,129,178$ and $219 \mathrm{~kg}$ for $\beta_{0}=0.01,0.05,0.1$ and 0.2 , respectively. Section 6.1 of this paper will show that transfers from above to below the equatorial plane and vice versa are possible and come at the cost of an almost negligible SEP propellant consumption. 
$h_{0}=35 \mathrm{~km}$

$\beta_{0}=0.01$

$\beta_{0}=0.1$
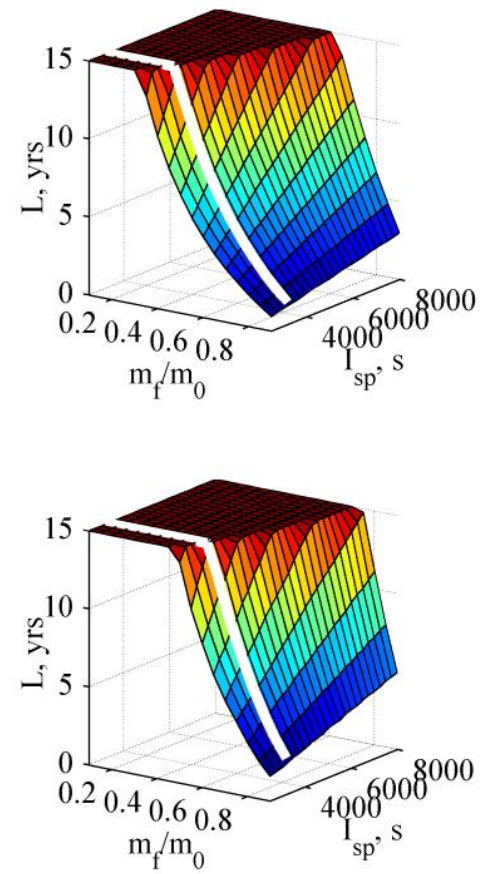

$\beta_{0}=0.2$

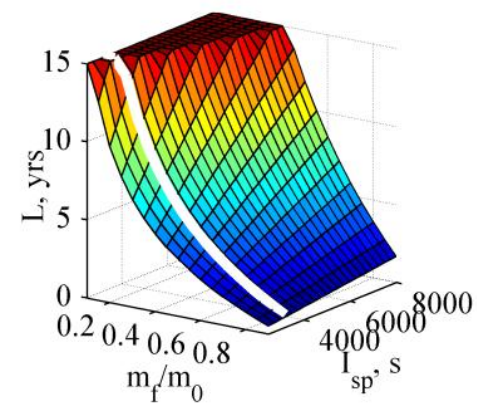

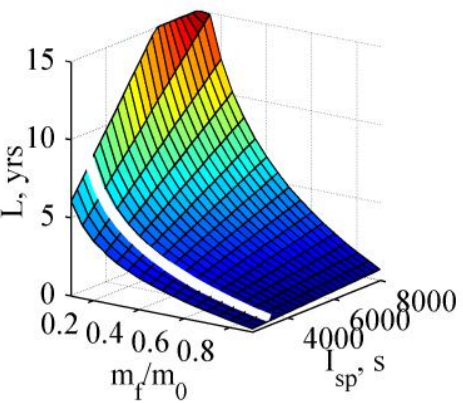
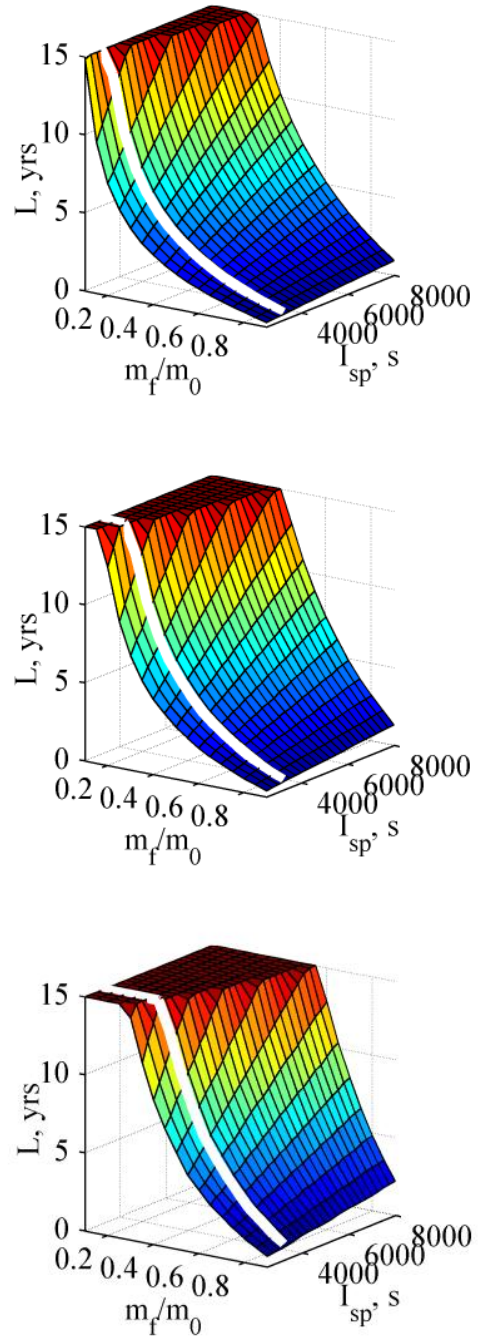

$h_{0}=150 \mathrm{~km}$
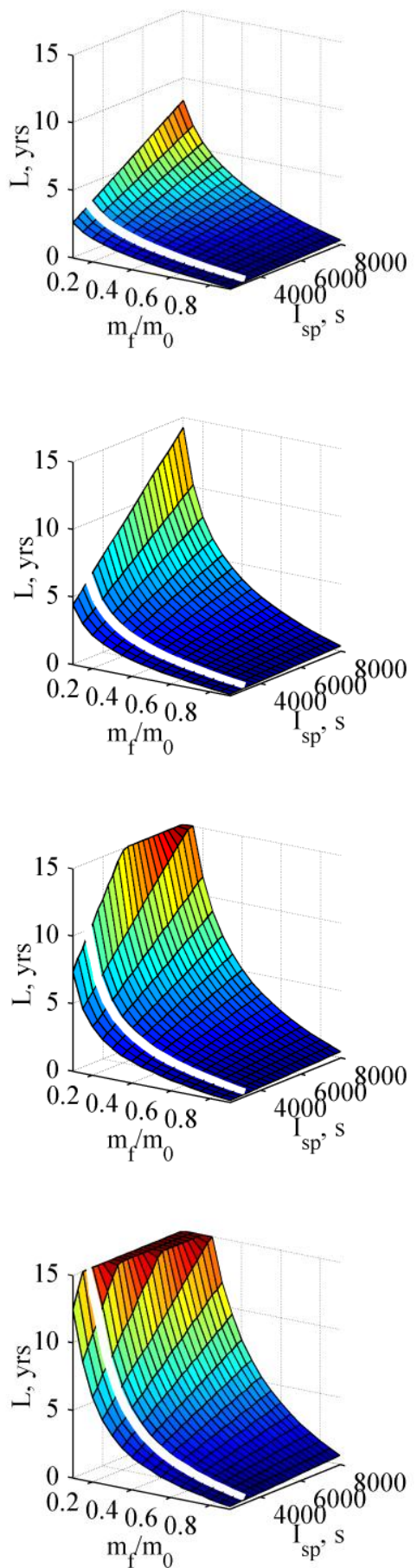

Fig. 8: Displaced geostationary orbits maintained with hybrid low-thrust control: mission time $L$ (maximum of 15 years) as a function of the specific impulse $I_{s p}$ (where the line indicates a currently feasible specific impulse of $3200 \mathrm{~s}$ ) and the mass fraction $m_{f} / m_{0}$, for different values of the solar sail lightness number $\beta_{0}$ and the initial displacement distance $h_{0}$. 
While the results in Fig. 7 only hold for a mission of 1 year, it is interesting to investigate whether hybrid propulsion can enable missions lasting as long as current geostationary missions. Fig. 2 already showed that SEP control is unable to do so. Extending the mission lifetime for hybrid low-thrust control results in the graphs shown in Fig. 8 which include the seasonal transfer to optimize the performance of the displaced geostationary orbit. Again, an arbitrary initial mass can be assumed and the three displacement distances of Section 2 are considered. The notation $h_{0}$ is used rather than $h$ to indicate that the spacecraft starts at a particular displacement (always above the equatorial plane for the results in Fig. 8) but is transferred between displacements above and below the equatorial plane during its lifetime. Note that all results neglect the effects of eclipses on the performance of the solar sail. For the (displaced) GEO, eclipses only occur for a short period per day around the equinoxes. It is assumed that increased SEP thrust can compensate for the absence of thrust from the solar sail during these brief periods.

Comparing Fig. 2 with Fig. 8 shows a dramatic improvement of the lifetime for hybrid low-thrust control compared to pure SEP control. Considering a $35 \mathrm{~km}$ displaced orbit, a mass fraction of 0.5 and a specific impulse of $3200 \mathrm{~s}$ shows an increase from 3.7 years for SEP control to 4.7, 9.7, >15 and >15 years for $\beta_{0}=0.01,0.05,0.1$ and 0.2 , respectively. Similarly, the lifetime for a $150 \mathrm{~km}$ displaced orbit is increased from 10 months to $1.9 \sim 2.2$ years, depending on the value for $\beta_{0}$. All in all, for hybrid low-thrust control, lifetimes of $10 \sim 15$ years come into reach for the smallest displacement, while reasonable lifetimes are obtained for the larger displacements.

\subsection{Perturbations due to non-uniform Earth gravity field}

Up to this point, the analyses for the displaced GEO have assumed a radially symmetrical mass distribution for the Earth. However, it is well known that the Earth's actual gravity field does not abide by this simple gravitational law, since the mass density distribution changes in North-South and East-West directions. Spacecraft in geostationary orbit are heavily affected by this, which requires costly station keeping maneuvers. A similar effect can be expected for the displaced GEO. This section will therefore investigate the influence of the non-uniform Earth's gravity field on the lifetimes depicted in Fig. 2 and Fig. 8 .

For this, the two most dominant perturbing accelerations will be considered, namely the so-called $J_{2}$ term (or Earth's oblateness) and the $J_{2,2}$ term. While the first term considers deviations of the Earth's mass density distribution in North-South direction, the second term is considered with deviations in East-West direction. For most spacecraft orbiting the Earth the effect of the latter will average out over periods longer than a day, but because a (displaced) GEO spacecraft is constantly located above the same point on Earth, it experiences a constant acceleration from the $J_{2,2}$ term and is therefore strongly perturbed.

Using a spherical reference frame with $r$ the distance from the center of the Earth, $\lambda$ the geographical longitude and $\phi$ the geocentric latitude, the perturbing accelerations due to the $J_{2}$ term can be derived from Ref. [30] as :

$$
\begin{aligned}
& a_{J_{2}, r}=\frac{3}{2} \mu J_{2} \frac{R_{E}^{2}}{r_{G E O}^{4}}\left(3 \sin ^{2} \phi-1\right) \\
& a_{J_{2}, \lambda}=0 \\
& a_{J_{2}, \phi}=-\frac{3}{2} \mu J_{2} \frac{R_{E}^{2}}{r_{G E O}^{4}} \sin 2 \phi
\end{aligned}
$$


with $R_{E}$ the radius of the Earth. Eq. (16) shows that, while the $J_{2}$ perturbing acceleration for a GEO spacecraft is purely radial, for the displaced GEO a non-zero term in $\phi$ direction exists.

Similarly, for the $J_{2,2}$ term the accelerations are given by:

$$
\begin{aligned}
& a_{J_{2,2}, r}=9 \mu J_{2,2} \frac{R_{E}^{2}}{r_{G E O}^{4}} \cos ^{2} \phi \cos 2\left(\lambda-\lambda_{2,2}\right) \\
& a_{J_{2,2}, \lambda}=6 \mu J_{2,2} \frac{R_{E}^{2}}{r_{G E O}^{4}} \cos \phi \sin 2\left(\lambda-\lambda_{2,2}\right) \\
& a_{J_{2,2}, \phi}=3 \mu J_{2,2} \frac{R_{E}^{2}}{r_{G E O}^{4}} \sin 2 \phi \cos 2\left(\lambda-\lambda_{2,2}\right)
\end{aligned}
$$

with $\lambda_{2,2}$ a coefficient related to the $J_{2,2}$ term. The accelerations in Eq. (16) and (17) can be added and transformed to the reference frame defined in Fig. 3. To determine the effect of the $J_{2}$ and $J_{2,2}$ terms on the performance of SEP and hybrid low-thrust propulsion for the displaced GEO, the resulting accelerations in $x_{E}, y_{E}$ and $z_{E}$ direction, denoted by $a_{J, x_{E}}, a_{J, y_{E}}$ and $a_{J, z_{E}}$, respectively, should be added to Eq. (6). This results in:

$$
\begin{aligned}
& a_{S E P, x_{E}}=a_{J, x_{E}} \quad-\beta_{0} \frac{m_{0}}{m} \frac{\mu_{S}}{r_{s}^{2}}\left(\cos \psi \sin \alpha_{s} \sin \delta_{s}+\sin \psi \cos \alpha_{s}\right)^{2} \sin \alpha_{s} \sin \delta_{s} \\
& a_{S E P, y_{E}}=a_{J, y_{E}} \quad-\beta_{0} \frac{m_{0}}{m} \frac{\mu_{S}}{r_{s}^{2}}\left(\cos \psi \sin \alpha_{s} \sin \delta_{s}+\sin \psi \cos \alpha_{s}\right)^{2} \sin \alpha_{s} \cos \delta_{s} \\
& a_{S E P, z_{E}}=a_{J, z_{E}}+\frac{\mu h}{r_{G E O}^{3}}-\beta_{0} \frac{m_{0}}{m} \frac{\mu_{S}}{r_{s}^{2}}\left(\cos \psi \sin \alpha_{s} \sin \delta_{s}+\sin \psi \cos \alpha_{s}\right)^{2} \cos \alpha_{s}
\end{aligned}
$$

Eq. (18) shows that the minimization problem in Eq. (7) still holds: for a particular instant of time, the acceleration required from the SEP system can be minimized by finding the optimal solar sail pitch and yaw angles. However, because the additional terms $a_{J, x_{E}}$ and $a_{J, y_{E}}$ are not constant along the displaced GEO, the optimal sail yaw angle is also no longer constant. Therefore, applying the approach of Eq. (8) to solve for the optimum solar sail pitch and yaw angles would require a system of nonlinear equations to be solved rather than the single expression in Eq. (12). That is why the minimization problem is solved using a sequential quadratic programming (SQP) method implemented in the MATLAB ${ }^{\circledR}$ function fmincon [31]. This function allows to define the bounds for the sail pitch angle $\alpha_{s}$ as shown in Fig. 5 and include a constraint to ensure $\left(\hat{\mathbf{n}} \cdot \hat{\mathbf{r}}_{s}\right) \geq 0$. As for the non-perturbed case, the displaced GEO is discretized into nodes with a time interval of $\Delta t=0.025$ days and at each node the minimization problem is solved.

The results for a spacecraft positioned in a $35 \mathrm{~km}$ displaced GEO at a longitude of $\lambda=0$ and with $I_{s p}=3200 \mathrm{~s}$ are provided in Fig. 9 and Fig. 10. In Fig. 9 the solar sail pitch and yaw angles are depicted to show their variation over one orbit due to the constantly changing $a_{J, x_{E}}$ and $a_{J, y_{E}}$ terms in Eq. (18). The actual influence on the spacecraft lifetime is shown in Fig. 10, which provides the loss in lifetime in percentage of the nominal, i.e. the unperturbed lifetime. The figure shows that the $J_{2}$ and $J_{2,2}$ terms have a small effect on the lifetime, which becomes almost negligible for the smaller values of the solar sail lightness number. For instance, for $\beta_{0}=0.01$ the spacecraft reaches a lifetime of 15 years at a mass fraction of approximately 0.16 . The corresponding percentage loss is 0.26 percent or 14 days. Note that the oscillating behavior for the case of hybrid low-thrust propulsion is introduced by the influence of the 
seasonally changing Sun-sail line. Similar results can be obtained for the larger displacements of 75 and $150 \mathrm{~km}$ since the magnitude of the perturbing acceleration does not change significantly.

Note that, besides the perturbing accelerations due to the $J_{2}$ and $J_{2,2}$ terms more perturbing accelerations act on a spacecraft in the displaced GEO, including third body perturbations from the Sun and the Moon. Other issues that can affect the performance of the hybrid propulsion system are solar sail and solar array degradation. It is expected, however, that these phenomena will have an even smaller influence on the lifetime of a spacecraft in the displaced GEO than the $J_{2}$ and $J_{2,2}$ terms and are therefore neglected in this paper.

a)

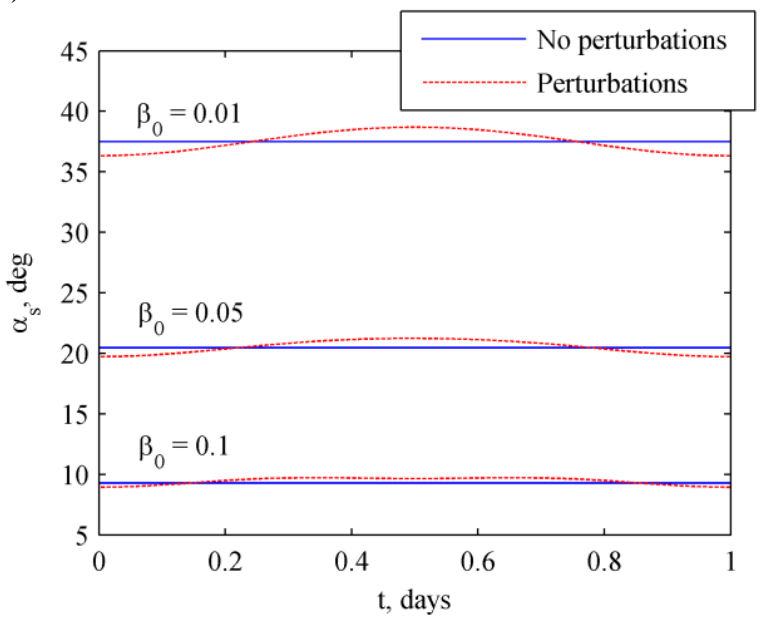

b)

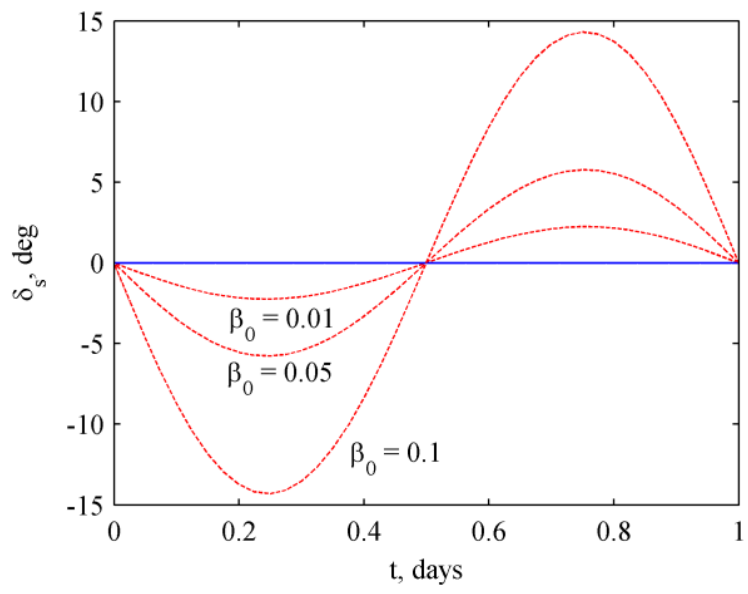

Fig. 9 Optimum solar sail pitch (a) and yaw (b) angles over one orbital period in winter when accounting for the perturbations due to the $J_{2}$ and $J_{2,2}$ terms of the Earth's gravity field for a spacecraft located at 0 deg longitude in a $35 \mathrm{~km}$ displaced GEO and for different values of the solar sail lightness number $\beta_{0}$.

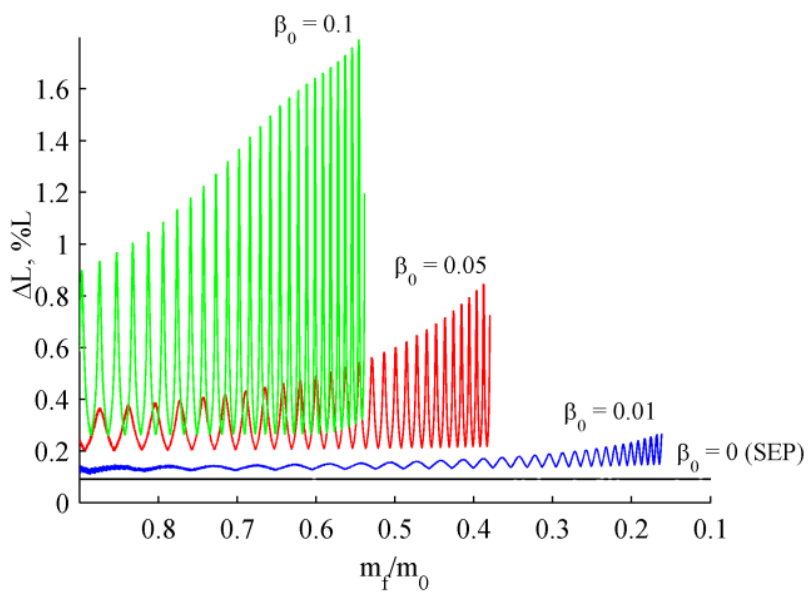

Fig. 10 Loss in mission lifetime as percentage of the nominal mission lifetime when accounting for the perturbations due to the $J_{2}$ and $J_{2,2}$ terms of the Earth's gravity field for a spacecraft located at 0 deg longitude in a $35 \mathrm{~km}$ displaced GEO and for different values of the solar sail lightness number $\beta_{0}$. 


\section{Mass budget}

The results in Fig. 2 and Fig. 8 provide the performance of both SEP and hybrid low-thrust control in terms of propellant consumption. However, the goal of the mission is to maximize the lifetime of a spacecraft while carrying a payload. It should therefore be investigated whether the mass fractions and specific impulses of Fig. 2 and Fig. 8 allow for a payload to be carried during the lifetimes shown in those figures. For this, the mass budget of the SEP and hybrid low-thrust controlled spacecraft are investigated. In this paper, the mass budget is based on what is proposed in Ref. [28]:

$m_{0}=m_{\text {pay }}+m_{\text {prop }}+m_{\text {tank }}+m_{S E P}+m_{P}+m_{\text {gimbal }}+m_{s}$

with $m_{0}$ the initial mass, $m_{\text {pay }}$ the payload mass and $m_{\text {prop }}$ the propellant mass that follows from the initial mass and the mass at lifetime $L$. Note that for the analysis in this section once again a radially symmetrical mass distribution for the Earth is assumed. $m_{\text {tank }}$ is the mass of the tanks required to store the propellant, which is given through $m_{\text {tank }}=0.1 m_{\text {prop }}$ [32], and $m_{S E P}$ is the mass of the SEP thruster. The SEP thruster mass is a function of the maximum power required by the SEP subsystem, $P_{S E P \text {, max }}$, which on its own is a function of the maximum thrust required during the mission, $T_{\max }$ :

$m_{S E P}=k_{S E P} P_{S E P, \max }$
$P_{S E P, \max }=\frac{T_{\max } I_{s p} g_{0}}{2 \eta_{S E P}}$

with $k_{S E P}=20 \mathrm{~kg} / \mathrm{kW}$ [33] the specific performance of the SEP thruster and $\eta_{\text {SEP }}=0.7$ [34] its efficiency. Subsequently, $m_{P}$ is the mass of the system that provides electrical energy to the SEP thruster. In case of SEP control a solar array with mass $m_{P}=k_{S A} P_{S E P, \max }$ is assumed with $k_{S A}=1 / 45 \mathrm{~kg} / \mathrm{W}$ the specific performance of the solar array [33]. In case of hybrid low-thrust control it is assumed that part of the solar sail is covered with thin film solar cells to provide the electrical power to the SEP system. The required area covered with solar cells can be computed from:

$A_{T F}=\frac{P_{S E P, \max }}{W \eta_{T F}} \cos \gamma_{S E P, \max }$

with $W=1367 \mathrm{~W} / \mathrm{m}^{2}$ the energy flux density of the Sun at $1 \mathrm{AU}, \eta_{T F}=0.05$ the efficiency of the thin film and $\gamma_{S E P_{\max }}$ the angle between the Sun-sail line, $\hat{\mathbf{r}}_{s}$, and the solar sail acceleration vector, $\hat{\mathbf{n}}$, when $T=T_{\max }$, see also Fig. 4 and Fig. 5. From this area the mass of the thin film $m_{P}=\sigma_{T F} A_{T F}$ can be computed with $\sigma_{T F}=100 \mathrm{~g} / \mathrm{m}^{2}$ [35]. Note that the influence of the thin film solar cells on the performance of hybrid lowthrust propulsion is neglected in this paper. Finally, $m_{\text {gimbal }}=0.3 m_{S E P}$ [32] is the mass of a gimbal that ensures that the solar sail and SEP thruster can steer independently from one another and $m_{s}$ is the mass of the sail that can be computed through $m_{s}=\sigma_{s} A_{s}$ with $\sigma_{s}=5 \mathrm{~g} / \mathrm{m}^{2}$ [36-37] the mass per unit area of the solar sail and $A_{s}$ the sail area, which is given by:

$$
A_{s}=\frac{\beta_{0} m_{0}}{\sigma^{*}}+A_{T F}
$$

Clearly, for an SEP controlled spacecraft, both $m_{\text {gimbal }}$ and $m_{s}$ are set to zero. At a given time and for a given specific impulse, the only unknowns for computing the payload mass are the initial mass and the maximum thrust required during the mission, which are related as the initial mass is bounded by the 
maximum available SEP thrust, $T_{\max }$. For SEP control, this maximum thrust occurs at $t=t_{0}$ causing $T_{\max }=T_{0}$. With the required acceleration to maintain the displaced geostationary orbit, $a$, given for a particular displacement distance, the maximum initial mass equals $m_{0, \max }=T_{0} / a$. For hybrid low-thrust control, the maximum thrust does not necessarily occur at $t=t_{0}$, but can also occur in autumn (when the seasonal transfer is taken into account as is done in this section) as shown in Fig. 7b. The resulting maximum initial masses for both SEP and hybrid low-thrust control are shown in Fig. 11 as a function of the maximum thrust magnitude and for each of the displacement distances used so far, for different sail lightness numbers and for a specific impulse of $3200 \mathrm{~s}$. Also a reference thrust magnitude of $0.2 \mathrm{~N}$ is indicated. The figure shows that for this reference thrust magnitude and SEP control, maximum initial masses of 1074 and $251 \mathrm{~kg}$ are possible for displacement distances of 35 and $150 \mathrm{~km}$, respectively. These initial masses increase by a factor 1.05 to 2.7 for hybrid low-thrust control, depending on the sail lightness number and the displacement distance. This is due to the reduced required SEP thrust magnitude for hybrid low-thrust propulsion compared to pure SEP control, which was already demonstrated in Fig. $7 \mathrm{~b}$.

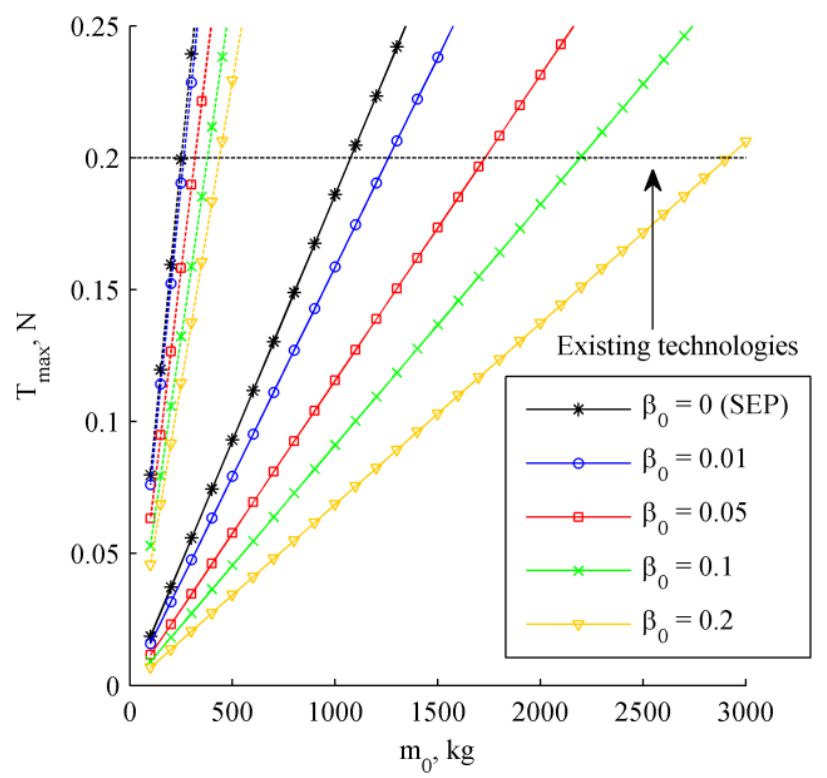

Fig. 11: Maximum initial mass as a function of the maximum thrust magnitude for a $35 \mathrm{~km}$ (solid lines) and $150 \mathrm{~km}$ (dashed lines) displaced geostationary orbit for different values of the sail lightness number $\beta_{0}$ and for $I_{s p}=3200 \mathrm{~s}$.

Using the maximum initial masses corresponding to a maximum thrust magnitude of $0.2 \mathrm{~N}$ in Fig. 11, the payload masses and lifetimes as depicted in Fig. 12 can be obtained. As a reference also the performance in terms of propellant consumption, as shown in Fig. 2 and Fig. 8, is depicted. The figure immediately shows that certain mass fractions considered in Fig. 2 and Fig. 8 do not allow for a payload mass to be carried on board the spacecraft. For example, for a $35 \mathrm{~km}$ displaced SEP controlled orbit and a mass fraction of 0.1 , a lifetime of 12.3 years can be obtained from a propellant consumption point of view. However, looking at the corresponding payload mass, it becomes clear that this mass fraction does not allow for a payload, simply because the propellant mass and the mass of the tanks containing the propellant become too large. Note that the payload masses in Fig. 12 can be increased when a larger maximum thrust magnitude and therefore a larger initial mass is allowed. However, this will not increase the maximum lifetime (i.e. the time at which no payload mass remains) as all mass components scale linearly with the initial mass or equivalently with the maximum thrust magnitude. Non-zero payload 
masses for longer lifetimes become possible when tuning spacecraft design parameters such as $k_{S E P}, \eta_{S E P}$, $k_{S A}, \sigma_{T F}, \eta_{T F}$ and $\sigma_{s}$.

Overall, Fig. 12 shows that in almost all cases hybrid low-thrust control outperforms SEP control. Only for the largest value of $\beta_{0}$ and a displacement of $150 \mathrm{~km}$, the large required sail area (and with that the sail mass) becomes a disadvantage. Furthermore, the figure shows that only hybrid low-thrust control allows lifetimes of current geostationary spacecraft of $10 \sim 15$ years while still enabling a considerable payload to be taken onboard. For example, for a $35 \mathrm{~km}$ displaced orbit, a sail lightness number of 0.1 and an initial mass of $2193 \mathrm{~kg}$, payload masses of $487 \mathrm{~kg}$ and $255 \mathrm{~kg}$ can be maintained in the displaced geostationary orbit for 10 and 15 years, respectively.

a)

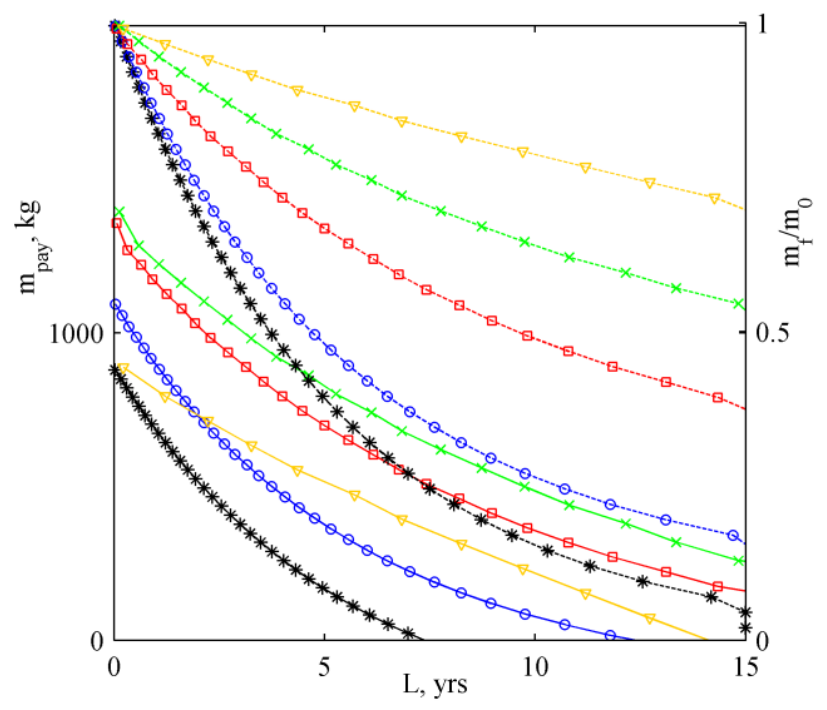

b)

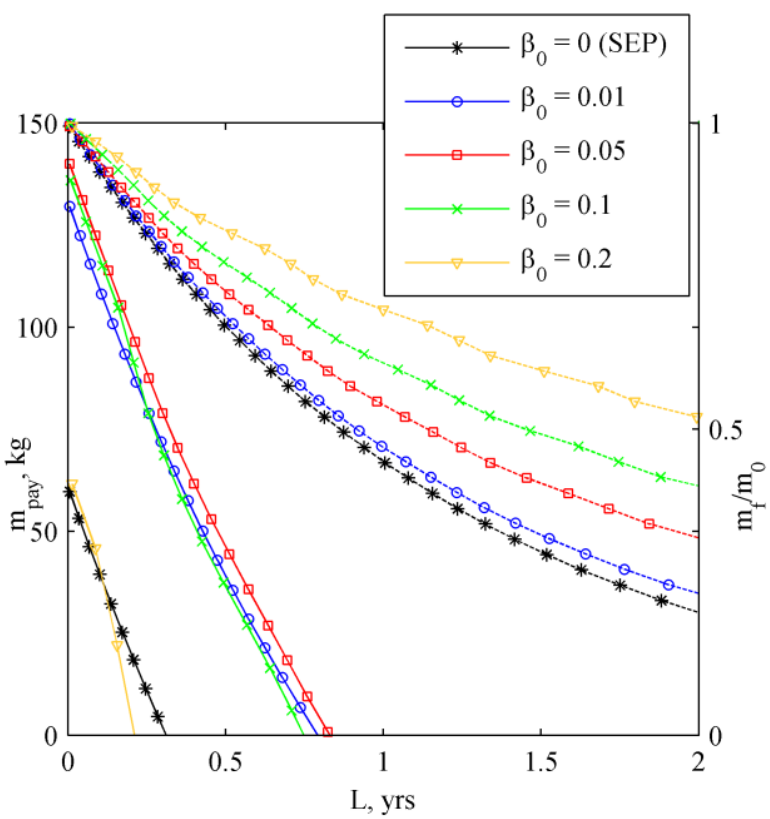

Fig. 12: Payload mass (solid lines) and mass fraction (dashed lines) as a function of the lifetime for a $35 \mathrm{~km}$ (a) and $150 \mathrm{~km}$ (b) displaced geostationary orbit for different values of the sail lightness number $\beta_{0}$ and for $I_{s p}=3200$.

Although the performance for a $35 \mathrm{~km}$ displaced orbit is highly promising, the performance of higher displaced orbits is not. Both the lifetime and the payload mass decrease significantly when larger displacements are considered. However, the performance of these larger displacements improves significantly if an increase in the maximum thrust magnitude is allowed. To show this improvement, a maximum thrust level of $1 \mathrm{~N}$ is assumed, which is considered reasonable for next generation SEP systems. Subsequently, requiring a payload mass of at least $200 \mathrm{~kg}$, the results in Table 1 can be found for a displacement of $150 \mathrm{~km}$ and a specific impulse of $3200 \mathrm{~s}$. Table 1 shows that a payload mass of $200 \mathrm{~kg}$ is indeed possible for reasonable values for the initial mass. The lifetime is, however, still rather short, just over 0.5 year. It can therefore be concluded that a $150 \mathrm{~km}$ displaced geostationary orbit is feasible using hybrid low-thrust control, be it for rather short periods of time. $150 \mathrm{~km}$ displaced geostationary orbits are therefore perfect for the concept of customized service using a mobile displaced geostationary platform. Then, the displaced geostationary orbit is only maintained for a relatively short period of time to provide additional coverage when needed (e.g. during major sporting events such as the Olympics or World Cup) and is transferred into a Keplerian parking orbit when inoperative. With only hours or days of coverage 
needed, the $150 \mathrm{~km}$ displaced geostationary orbit can transform its rather short lifetime into multiple smaller missions extended over a much longer lifetime. To show the feasibility of this concept, the next section will investigate the transfer that is required to transfer the spacecraft from and to the Keplerian parking orbit.

Table 1: Lifetime $L$, payload mass $m_{\text {pay }}$ and initial mass $m_{0}$ for a $150 \mathrm{~km}$ displaced geostationary orbit allowing $T_{\max } \leq 1.0 \mathrm{~N}$, requiring $m_{p a y} \geq 200 \mathrm{~kg}$ and using $I_{s p}=3200 \mathrm{~s}$.

\begin{tabular}{lccc}
\hline & $\beta_{0}=0.01$ & $\beta_{0}=0.05$ & $\beta_{0}=0.1$ \\
\hline$L, \mathrm{yrs}$ & 0.48 & 0.54 & 0.47 \\
$m_{\text {pay }}, \mathrm{kg}$ & 206 & 200 & 202 \\
$T_{\max }, \mathrm{N}$ & 0.99 & 0.98 & 0.98 \\
$m_{0}, \mathrm{~kg}$ & 1300 & 1550 & 1850 \\
\hline
\end{tabular}

\section{Transfer orbits}

In the previous sections two types of transfers where mentioned to improve the performance of hybrid low-thrust control while maintaining the displaced geostationary orbit. This section will investigate these transfers.

\subsection{Seasonal transfer}

As mentioned in Section 4, the obliquity of the ecliptic causes hybrid low-thrust control for displaced geostationary orbits to perform best when a spacecraft is displaced above the equatorial plane in winter and below the equatorial plane in summer. To accomplish this, the spacecraft will have to be transferred from above the equatorial plane to below the equatorial plane and vice versa twice per year: once in spring (above to below) and once in autumn (below to above). This section will optimize this transfer for the SEP propellant consumption, which implies solving the accompanying optimal control problem. An optimal control problem is to find a state history $\mathbf{x}(t) \in \mathbb{R}^{n_{x}}$ and a control history $\mathbf{u}(t) \in \mathbb{R}^{n_{u}}, t \in\left[t_{0}, t_{f}\right]$, subject to the dynamics:

$\dot{\mathbf{x}}(t)=\mathbf{f}(\mathbf{x}(t), \mathbf{u}(t), t)$

that minimize the cost function:

$$
J=\varphi\left(\mathbf{x}_{0}, \mathbf{x}_{f}, t_{0}, t_{f}\right)+\int_{t_{0}}^{t_{f}} L(\mathbf{x}(t), \mathbf{u}(t), t) d t
$$

and satisfy the constraints $\mathbf{c}(\mathbf{x}, \mathbf{u}, t) \leq 0$. These constraints can include event constraints on the initial and final states and time, bounds on the state variables, control variables and time and path constraints. The first term on the right hand side of Eq. (20) is the endpoint (Mayer-type) cost function, which is only a function of the initial and final states and initial and final time, while the second term is the Lagrange cost function which is a function of time. To solve this optimal control problem the open source tool PSOPT is applied [38]. PSOPT implements a direct pseudospectral method to solve the optimal control problem. By discretizing the time interval into a finite number of nodes, the infinite dimensional optimal control problem is transformed into a finite dimension non-linear programming (NLP) problem. Pseudospectral methods use Legendre or Chebyshev polynomials to approximate and interpolate the time dependent variables at the nodes. The advantage of using pseudospectral methods is that the derivatives of the state 
functions at the nodes are computed by matrix multiplication only and that any integral associated with the problem is approximated using well known Gauss quadrature rules.

To optimize the seasonal transfer for the SEP propellant consumption, the cost function equals:

$J=-m_{f}$

with $m_{f}$ the final mass of the spacecraft. The seasonal transfer is described using a spherical reference frame $C(r, \theta, \phi)$ centered at the Earth, see Fig. 13. The in-plane angle $\theta$ is measured in counter clockwise direction from the $x_{C}$ axis that coincides with the start of the transfer (i.e. for $t=0, \theta=0$ ) and the out-ofplane angle $\phi$ is measured from the $\left(x_{C}, y_{C}\right)$ plane that is parallel to the equatorial plane. For an SEP controlled spacecraft the state vector at any point in the trajectory then becomes:

$\mathbf{x}=\left[\begin{array}{lllllll}r & \theta & \phi & V_{r} & V_{\theta} & V_{\phi} & m\end{array}\right]$

with $V_{r}, V_{\theta}$ and $V_{\phi}$ the velocity in $r, \theta$ and $\phi$ direction, respectively and $m$ the mass of the spacecraft.

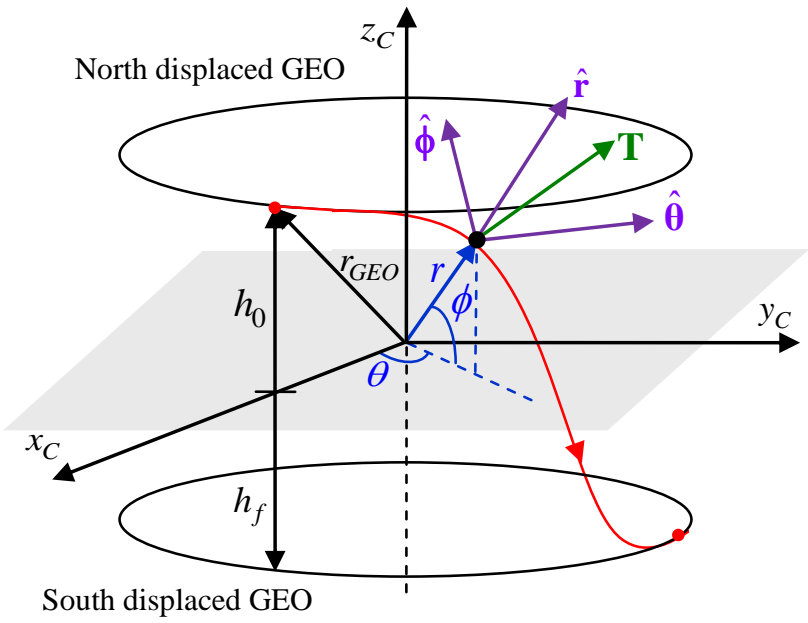

Fig. 13: Definition of spherical reference frame to describe the seasonal transfer.

With the transfer starting and ending in a displaced geostationary orbit the initial, $\mathbf{x}_{0}$, and final, $\mathbf{x}_{f}$, state vectors are given by:

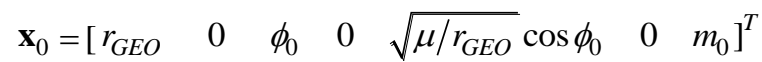

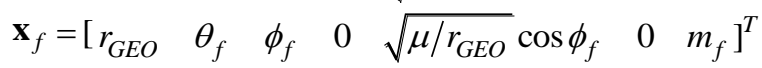

with the final mass $m_{f}$ free. The final in-plane angle $\theta_{f}$ is restricted to:

$\theta_{f}=\omega_{G E O} t_{f}$

with $\omega_{G E O}$ the angular velocity in the (displaced) geostationary orbit to ensure that the longitude of the spacecraft in the displaced geostationary orbit is unchanged after the transfer. Furthermore, $\phi_{0}=\sin ^{-1}\left(h_{0} / r_{G E O}\right)$ and $\phi_{f}=\sin ^{-1}\left(h_{f} / r_{G E O}\right)$. Correct signs for $h_{0}$ and $h_{f}$ will ensure correct signs for $\phi_{0}$ and $\phi_{f}$. 
Using a two-body model the equations that describe the motion of the spacecraft in the transfer become:

$$
\dot{\mathbf{x}}=\left(\begin{array}{c}
V_{r} \\
V_{\theta} /(r \cos \phi) \\
V_{\phi} / r \\
V_{\theta}^{2} / r+V_{\phi}^{2} / r-\mu / r^{2}+T_{r} / m \\
-V_{r} V_{\theta} / r+V_{\theta} V_{\phi} \tan \phi / r+T_{\theta} / m \\
-V_{r} V_{\phi} / r-V_{\theta}^{2} \tan \phi / r+T_{\phi} / m \\
-T /\left(I_{s p} g_{0}\right)
\end{array}\right)
$$

with $\mathbf{u}=\left[\begin{array}{lll}T_{r} & T_{\theta} & T_{\phi}\end{array}\right]$ the control vector consisting of the Cartesian components of the SEP thrust acceleration. Note that the Cartesian thrust components are used rather than two thrust angles and the thrust magnitude as these may give rise to ambiguities [39]. However, using Cartesian components requires the following path constraint:

$T=\sqrt{T_{r}^{2}+T_{\theta}^{2}+T_{\phi}^{2}} \leq T_{\max }$

Finally, the bounds on the state and control variables and the transfer time are set to:

$$
\begin{gathered}
\mathbf{x}_{L}=\left[\begin{array}{ccccccc}
R_{E}+100 & 0 & -0.5 \pi & -10 & -10 & -10 & 0
\end{array}\right]^{T} \\
\mathbf{x}_{U}=\left[\begin{array}{ccccccc}
R_{E}+100,000 & 4 \pi & 0.5 \pi & 10 & 10 & 10 & m_{0}
\end{array}\right]^{T} \\
\mathbf{u}_{L}=\left[\begin{array}{lllll}
-T_{\max } & -T_{\max } & -T_{\max }
\end{array}\right] \\
\mathbf{u}_{U}=\left[\begin{array}{cccc}
T_{\max } & T_{\max } & T_{\max }
\end{array}\right] \\
t_{L}=0 \\
t_{U}=1 \text { day }
\end{gathered}
$$

with $R_{E}$ the radius of the Earth and $T_{\max }$ the maximum allowed thrust magnitude. Distances are provided in $\mathrm{km}$, velocities in $\mathrm{km} / \mathrm{s}$ and angles in radians. Eq. (21) shows that wide bounds are set on the state variables in order not to restrict the optimizer. For instance, the bounds on the components of the velocities are set far beyond the velocity in the displaced geostationary orbit and two full orbital revolutions are allowed for the transfer. Finally, a maximum transfer time of one day is assumed to limit a potential disruption in the downlink to Earth during the transfer.

PSOPT requires a first guess to initialize the optimization. To obtain this first guess, a shape-based approach is used in which the shape of the transfer is fixed and the required controls to perform that transfer are sought for. For this, the transfer is considered in a rotating reference frame that rotates with respect to an inertial frame at constant angular velocity equal to the angular velocity of the (displaced) geostationary orbit. Within this rotating frame, spacecraft in the displaced geostationary orbits are stationary. The transfer between the orbits is assumed to be the shortest path possible in this reference frame and a parabolic velocity profile is adopted to ensure zero velocities at the start and end of the transfer.

The results of the optimization in PSOPT are given in Table 2 with the corresponding thrust profiles in Fig. 14. A maximum thrust magnitude of $0.2 \mathrm{~N}$ is assumed leading to the use of the initial masses as determined in Fig. 11. To consider the worst case scenario, the initial masses corresponding to $\beta_{0}=0.2$ are selected. The table shows a relatively worse performance for smaller displacements which can be explained by the higher initial mass that can be put in the orbit without exceeding the maximum thrust 
level of $0.2 \mathrm{~N}$ while in-orbit. Table 2 furthermore shows that almost negligible amounts of propellant are needed to perform the seasonal transfer, which justifies the usage of this switch in Section 4 to improve the performance of hybrid low-thrust control. The reason for the extremely small amounts of propellant needed for the seasonal transfer can be found in the fact that the spacecraft falls into a Keplerian orbit when switching off the thrust in the displaced geostationary orbit. The start of this Keplerian orbit coincides with the apogee, while the perigee almost touches the displaced geostationary orbit on the other side of the equatorial plane [2]. Thus, only a tiny thrust force in the form of a bang-off-bang control is needed to overcome the small offset between the perigee of the Keplerian transfer orbit and the displaced geostationary orbit.

Table 2: Required propellant mass for optimized seasonal transfer.

\begin{tabular}{lcc}
\hline$h_{0}, \mathrm{~km}$ & $m_{0}, \mathrm{~kg}$ & $m_{\text {prop }}, \mathrm{g}$ \\
\hline \pm 35 & 2912 & 2.60 \\
\pm 75 & 1020 & 0.96 \\
\pm 150 & 436 & 0.66 \\
\hline
\end{tabular}

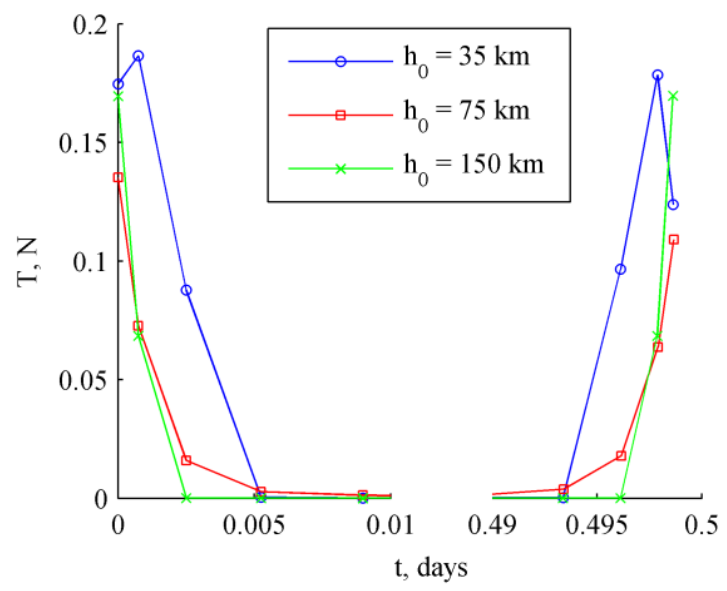

Fig. 14: Thrust profile for optimized seasonal transfer.

\subsection{Transfer from and to parking orbit}

In Section 5 the concept of customized service by using a mobile displaced geostationary platform was introduced. Then, the spacecraft is transferred into a displaced geostationary orbit for a relatively short period of time to deliver any required coverage and is transferred back into a Keplerian parking orbit when the geostationary coverage is no longer needed. This parking orbit and the transfer that are involved in this concept are depicted in Fig. 15. The parking orbit thus lies inside the geostationary orbit where the distance between the parking orbit and the geostationary orbit equals the absolute value of the displacement distance. In this way, the parking orbit is as close to the displaced geostationary orbit as possible without interfering with either the geostationary or the displaced geostationary orbit.

The investigation of this transfer is very similar to the method used for the seasonal transfer. The definition of the state and control variables is the same as are the equations of motion. Only the initial and final states differ. When the transfer from the parking orbit to the displaced geostationary orbit is considered, these become: 


$$
\begin{aligned}
& \mathbf{x}_{0}=\left[\begin{array}{lllllll}
r_{G E O}-|h| & 0 & 0 & 0 & \sqrt{\mu /\left(r_{G E O}-|h|\right)} & 0 & m_{0}
\end{array}\right]^{T} \\
& \mathbf{x}_{f}=\left[\begin{array}{lllllll}
r_{G E O} & \theta_{f} & \phi_{f} & 0 & \sqrt{\mu / r_{G E O}} \cos \phi_{f} & 0 & m_{f}
\end{array}\right]^{T}
\end{aligned}
$$

with the final in-plane angle $\theta_{f}$ and final mass $m_{f}$ free. Phasing between the parking orbit and the displaced geostationary orbit will have to ensure that the spacecraft is inserted into the displaced geostationary orbit at the correct longitude. Note that when the transfer from the displaced orbit to the parking orbit is considered, the initial condition equals Eq. (23) and the final condition becomes Eq. (22).

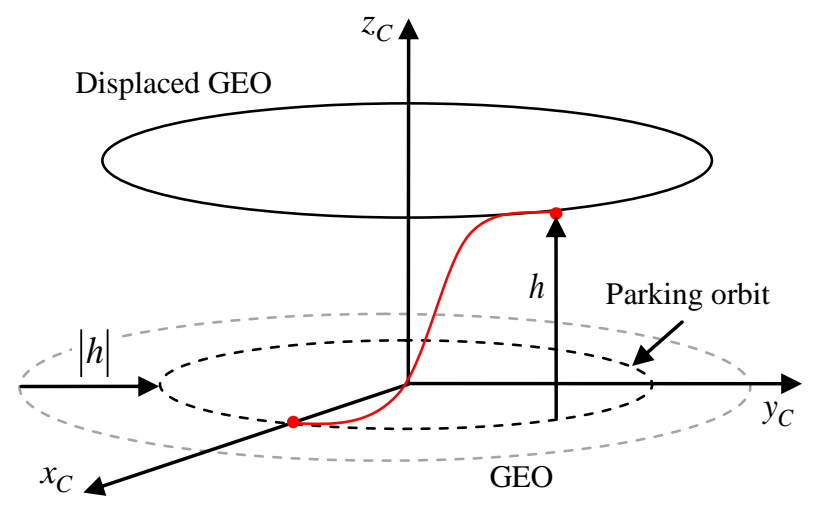

Fig. 15: Definition of parking orbit and transfer for customized geostationary service.

Also the optimization of the transfer is similar to the optimization of the seasonal transfer. The same objective function, bounds on the state and control variables and path constraint can be applied. Even the method to generate the initial guess is the same. The only slight difference is the fact that a somewhat larger transfer time is allowed.

The results of the optimization are shown in Table 3, Fig. 16 and Fig. 17. Table 3 shows that, although the required propellant is a factor 100 larger than for the seasonal transfer, the transfer still requires only modest propellant budgets. Fig. 14 and Fig. 15 furthermore show that the transfer from the parking orbit to the displaced orbit requires a different thrust profile than the transfer from the displaced orbit to the parking orbit. The three thrust arc profile is mainly observed for the higher displaced orbits, which suggests that the required inclination change to go from the parking to the displaced orbit cannot be achieved in a two thrust arc strategy, while this is possible for the transfer in the opposite direction.

Table 3: Required propellant mass in grams for the customized service transfer. a) Transfer from parking orbit to displaced geostationary orbit. b) Transfer from displaced geostationary orbit to parking orbit.

a)

\begin{tabular}{lcc}
\hline$h_{f}, \mathrm{~km}$ & $m_{0}, \mathrm{~kg}$ & $m_{\text {prop }}, \mathrm{g}$ \\
\hline \pm 35 & 2912 & 277.8 \\
\pm 75 & 1020 & 204.0 \\
\pm 150 & 436 & 173.7 \\
\hline
\end{tabular}

a) b)

\begin{tabular}{lcc}
\hline$h_{0}, \mathrm{~km}$ & $m_{0}, \mathrm{~kg}$ & $m_{\text {prop }}, \mathrm{g}$ \\
\hline \pm 35 & 2912 & 292.0 \\
\pm 75 & 1020 & 208.9 \\
\pm 150 & 436 & 176.1 \\
\hline
\end{tabular}

b) 

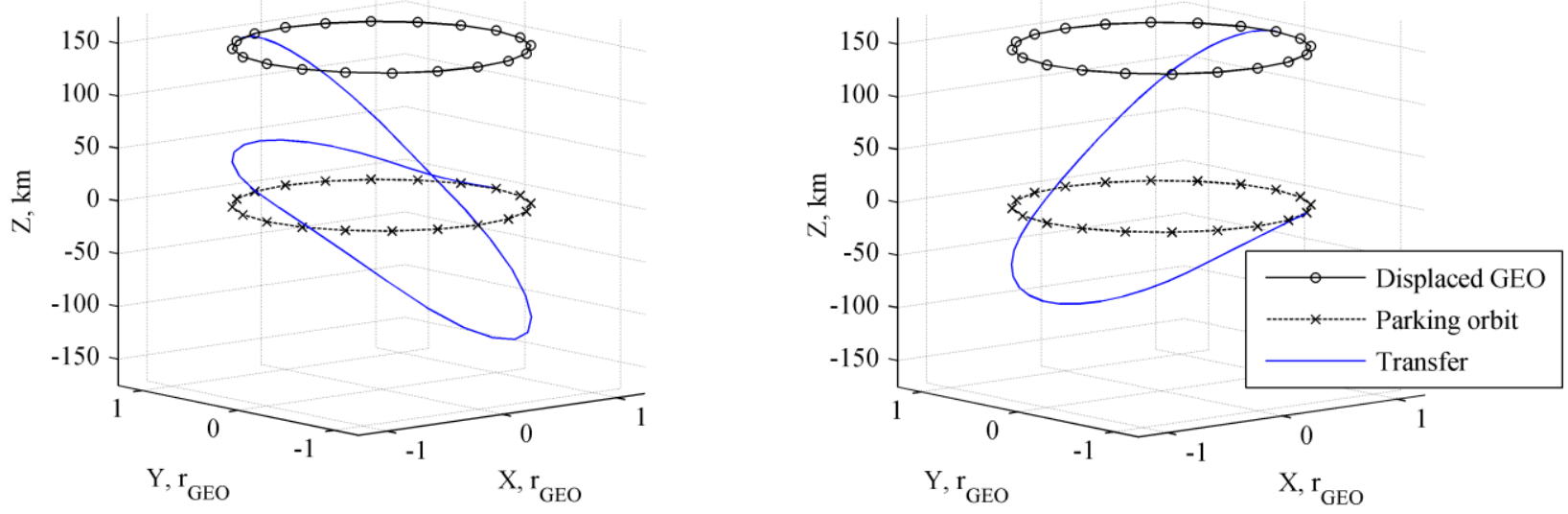

Fig. 16: Customized service transfer for a $150 \mathrm{~km}$ displaced geostationary orbit. a) Transfer from parking orbit to displaced geostationary orbit. b) Transfer from displaced geostationary orbit to parking orbit.

a)

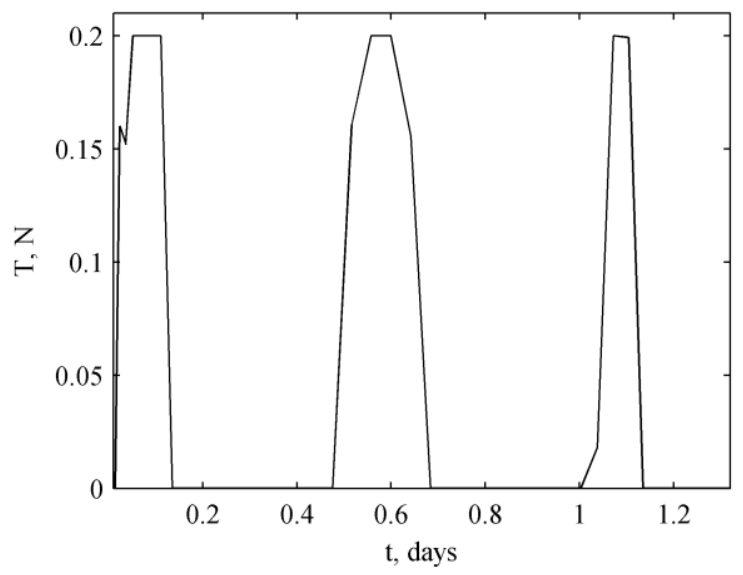

b)

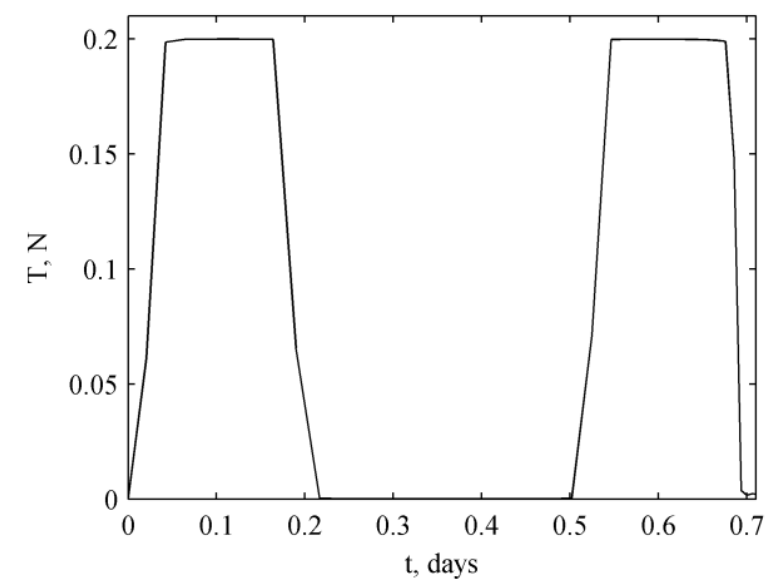

Fig. 17: Thrust profile for a customized service transfer for a $150 \mathrm{~km}$ displaced geostationary orbit. a) Transfer from parking orbit to displaced geostationary orbit. b) Transfer from displaced geostationary orbit to parking orbit.

\section{Conclusions}

In this paper geostationary orbits displaced above and below the equatorial plane have been proposed to increase the capacity of the geostationary ring that is starting to become congested. To maintain the orbit, two types of control have been suggested, Solar Electric Propulsion (SEP) control and hybrid low-thrust control. Both types of control have been optimized for the SEP propellant consumption, thereby maximizing the mission lifetime and/or payload mass. SEP control appeared to enable lifetimes of a few months in a $150 \mathrm{~km}$ displaced orbit to a few years in a $35 \mathrm{~km}$ displaced orbit, the minimum to rise above the geostationary station keeping box. However, investigating the spacecraft mass budget showed that only for small displacements reasonable payload masses of a few hundred kilograms could be maintained for a few years. By adding a solar sail to the SEP system, thereby creating hybrid low-thrust control, the demand on the SEP system could be lowered significantly while enabling a mission that is impossible using only solar sailing due to the obliquity of the ecliptic. An even better performance was obtained by 
alternating the displacement between above (autumn - spring) and below (spring - autumn) the equatorial plane during the year to make advantage of the seasonally changing Sun-sail line, introducing a so-called seasonal transfer. Optimizing this transfer for the SEP propellant consumption showed that this transfer comes almost for free. Employing this transfer showed that hybrid low-thrust control outperforms the pure SEP case both in terms of payload mass capacity and mission lifetime for all displacements considered. Hybrid low-thrust control provides lifetimes of $10 \sim 15$ years (equal to current geostationary missions) for a $35 \mathrm{~km}$ displaced orbit and for considerable payload masses of $255 \sim 487 \mathrm{~kg}$. Including the perturbing accelerations due to the $J_{2}$ and $J_{2,2}$ terms of the Earth's gravity field showed to have a small effect on this lifetime, which reduces to negligible values for small sail lightness numbers. Allowing a somewhat larger maximum thrust magnitude also resulted in reasonable payload masses of $200 \mathrm{~kg}$ for the higher displaced orbits, be it for relatively short periods of time. These orbits therefore appeared to be especially useful for the concept of customized service in which the spacecraft is only operative in the displaced orbit for relatively short periods of time (hours or days) to provide coverage when needed. When not operational, the spacecraft is transferred into a Keplerian parking orbit. Optimizing this transfer showed that only a modest propellant budget of approximately $200 \mathrm{~g}$ is required.

\section{Acknowledgments}

This work was funded by the European Research Council Advanced Investigator Grant - 227571: Visionary Space Systems: Orbital Dynamics at Extremes of Spacecraft Length-Scale. The author would like to thank Dr. Victor M. Becerra for providing the software tool PSOPT freely and for providing advice on its use.

\section{References}

[1] R. Jehn, A. Rossi, T. Flohrer, D. Navarro-Reyes, Reorbiting of satellites in high altitudes, Darmstadt, Germany, 2009.

[2] C.R. McInnes, Solar Sailing: Technology, Dynamics and Mission Applications, Springer-Praxis Books in Astronautical Engineering, Springer-Verlag, Berlin, 1999.

[3] C.R. McInnes, Dynamics, Stability, and Control of Displaced Non-Keplerian Orbits, Journal of Guidance, Control, and Dynamics, 21 (1998) 799-805.

[4] J.D. Biggs, C.R. McInnes, T. Waters, Control of Solar Sail Periodic Orbits in the Elliptic Three-Body Problem, Journal of Guidance, Control, and Dynamics, 32 (2009) 318-320.

[5] C.R. McInnes, The Existence And Stability Of Families Of Displaced Two-Body Orbits, Celestial Mechanics and Dynamical Astronomy, 67 (1997) 167-180.

[6] T.R. Spilker, Saturn Ring Observer, Acta Astronautica, 52 (2003) 259-265.

[7] T.J. Waters, C.R. McInnes, Periodic Orbits Above the Ecliptic in the Solar-Sail Restricted Three-Body Problem, Journal of Guidance, Control, and Dynamics, 30 (2007) 687-693.

[8] J. Simo, C.R. McInnes, Solar Sail Orbits at the Earth-Moon Libration Points, Communications in Nonlinear Science and Numerical Simulation, 14 (2009) 4191-4196.

[9] D.J. Grebow, M.T. Ozimek, K.C. Howell, Advanced Modeling of Optimal Low-Thrust Lunar PoleSitter Trajectories, Daejon, South Korea, 2009.

[10] J. Bookless, C.R. McInnes, Dynamics and Control of Displaced Periodic Orbits Using Solar-Sail Propulsion, Journal of Guidance, Control, and Dynamics, 29 (2006) 527-537.

[11] O. Mori, H. Sawada, R. Funase, T. Endo, M. Morimoto, T. Yamamoto, Y. Tsuda, Y. Kawakatsu, J. Kawaguchi, Development of First Solar Power Sail Demonstrator - IKAROS, Toulouse, France, 2009.

[12] K. Newton, "NASA's First Solar Sail Nano Sail-D Deploys in Low-Earth Orbit", available from: http://www.nasa.gov/centers/marshall/news/news/releases/2011/11-010.html, cited 24 January 2011.

[13] M. Macdonald, C.R. McInnes, Solar Sail Mission Applications and Future Advancement, New York, USA, 2010. 
[14] S. Baig, C.R. McInnes, Light-Levitated Geostationary Cylindrical Orbits are Feasible, Journal of Guidance, Control, and Dynamics, 33 (2010) 782-793.

[15] R. McKay, M. Macdonald, F. Bosquillon de Frescheville, M. Vasile, C. McInnes, J. Biggs, NonKeplerian Orbits Using Low Thrust, High ISP Propulsion Systems, IAC-09.C1.2.8, Daejeon, South Korea, 2009.

[16] M.D. Rayman, P.A. Chadbourne, J.S. Culwell, S.N. Williams, Mission Design for Deep Space 1: A Low-Thrust Technology Validation Mission, Acta Astronautica, 45 (1999) 381-388.

[17] G.D. Racca, A. Marini, L. Stagnaro, J. van Dooren, L. di Napoli, B.H. Foing, R. Lumb, J. Volp, J. Brinkmann, R. Grünagel, D. Estublier, E. Tremolizzo, M. McKay, O. Camino, J. Schoemaekers, M. Hechler, M. Khan, P. Rathsman, G. Andersson, K. Anflo, S. Berge, P. Bodin, A. Edfors, A. Hussain, J. Kugelberg, N. Larsson, B. Ljung, L. Meijer, A. Mörtsell, T. Nordebäck, S. Persson, F. Sjöberg, SMART-1 mission description and development status, Planetary and Space Sciences, 50 (2002) 1323-1337.

[18] J.R. Brophy, M.A. Etters, J. Gates, C.E. Garner, M. Klatte, C.J. Lo, M.G. Marcucci, S. Mikes, G. Pixler, B. Nakazono, Development and Testing of the Dawn Ion Propulsion System, AIAA-2006-4319, Sacramento, USA, 2006.

[19] G. Mengali, A.A. Quarta, Trajectory Design with Hybrid Low-Thrust Propulsion System, Journal of Guidance, Control, and Dynamics, 30 (2007) 419-426.

[20] G. Mengali, A.A. Quarta, Tradeoff Performance of Hybrid Low-Thrust Propulsion System, Journal of Spacecraft and Rockets, 44 (2007) 1263-1270.

[21] J. Simo, C.R. McInnes, Designing displaced lunar orbits using low-thrust propulsion, Journal of Guidance, Control, and Dynamics, 33 (2010) 259-265.

[22] S. Baig, C.R. McInnes, Artificial Three-Body Equilibria for Hybrid Low-Thrust Propulsion, Journal of Guidance, Control, and Dynamics, 31 (2008) 1644-1655.

[23] R.J. McKay, M. Macdonald, M. Vasile, F. Bosquillon de Frescheville, A Novel Interplanetary Communications Relay, AIAA-2010-7964, Toronto, Canada, 2010.

[24] M. Ceriotti, C.R. McInnes, Generation of Optimal Trajectories for Earth Hybrid Pole-Sitters, Journal of Guidance, Control, and Dynamics, 34 (2011).

[25] B.G. Evans, Satellite Communication Systems, 3rd Edition ed., The Institution of Engineering and Technology, London, UK, 1999.

[26] UNCOPUOS, Long-term sustainability of outer space activities, Preliminary reflections, Vienna, Austria, 2010.

[27] H. Kuninaka, K. Nishiyama, I. Funaki, T. Yamada, Y. Shimizu, J. Kawaguchi, Powered Flight of Electron Cyclotron Resonance Ion Engines on Hayabusa Explorer, Journal of Propulsion and Power, 23 (2007).

[28] M. Ceriotti, C. McInnes, A Near Term Pole-Sitter Using Hybrid Solar Sail Propulsion, New York, USA, 2010.

[29] A. Schneider, W. Sun, H. Schuff, The European Platform LUXOR for Small Communications Satellites, AIAA-2008-5441, San Diego, USA, 2008.

[30] D.A. Vallado, Fundamentals of Astrodynamics and Applications, 3rd Edition ed., Space Technology Library, New York, USA, 2007, pp. 536-548.

[31] M. Powell, A fast algorithm for nonlinearly constrained optimization calculations, in: G. Watson

(Ed.) Numerical Analysis, Springer Berlin / Heidelberg, 1978, pp. 144-157.

[32] R. Gershman, C. Seybold, Propulsion Trades for Space Science Missions, Acta Astronautica, 45 (1999) 541-548.

[33] J.R. Wertz, W.J. Larson, Space Mission Analysis and Design, 3rd Edition ed., Space Technology Library, Microcosm Press/Kluwer Academic Publishers, El Segundo, USA/London, UK, 1999.

[34] S. Kitamura, Y. Ohkawa, Y. Hayakawa, H. Yoshida, K. Miyazaki, Overview and research status of the JAXA 150-mN ion engine, Acta Astronautica, 61 (2007) 360-366.

[35] M. Leipold, M. Götz, Hybrid Photonic/Electric Propulsion, Kayser-Threde GmbH, Technical Report SOL4-TR-KTH-001, ESA contract No. 15334/01/NL/PA, Munich, Germany, 2002. 
[36] D.M. Murphy, T.W. Murphey, P.A. Gierow, Scalable solar-sail subsystem design considerations, American Inst. Aeronautics and Astronautics Inc., AIAA-2002-1703, Denver, USA, 2002.

[37] B. Dachwald, Optimal Solar Sail Trajectories for Missions to the Outer Solar System, AIAA-20045406, Providence, USA, 2004.

[38] V.M. Becerra, Solving complex optimal control problems at no cost with PSOPT, Yokohama, Japan, 2010, pp. 1391-1396.

[39] J.T. Betts, Practical Methods for Optimal Control Using Nonlinear Programming, Society for Industrial and Applied Mathematics (SIAM), Philadelphia, USA, 2001, pp. 150. 


\section{Jeannette Heiligers}

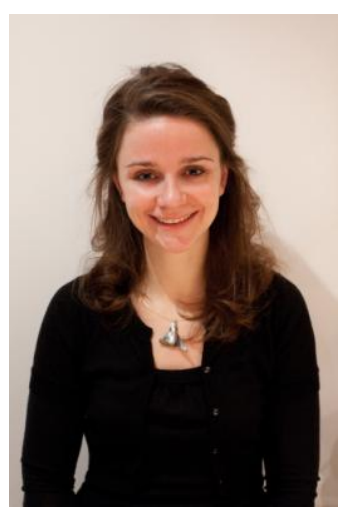

Jeannette Heiligers is a Ph.D. candidate at the Advanced Space Concepts Laboratory at the University of Strathclyde. She obtained her M.Sc. cum laude in Aerospace Engineering from Delft University of Technology, the Netherlands. Funded by the European Research Council, the PhD currently focuses on the orbital dynamics of non-Keplerian orbits using hybrid solar sail and solar electric propulsion and the optimization of transfers to and between non-Keplerian orbits. Previously, for her M.Sc., Jeannette worked on the multi-objective optimization of both high- and low-thrust trajectories for an asteroid sample return mission and she participated in the mission analysis for the European Student Moon Orbiter mission of the European Space Agency.

\section{Colin McInnes}

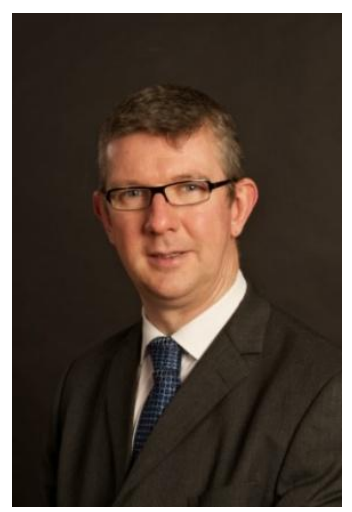

Colin McInnes is Director of the Advanced Space Concepts Laboratory at the University of Strathclyde. His work spans highly non-Keplerian orbits, orbital dynamics and mission applications for solar sails, spacecraft control using artificial potential field methods and is reported in over 100 journal papers. Recent work is exploring new approaches to spacecraft orbital dynamics at extremes of spacecraft lengthscale to underpin future space-derived products and services. McInnes has been the recipient of awards including the Royal Aeronautical Society Pardoe Space Award (2000), the Ackroyd Stuart Propulsion Prize (2003) and a Leonov medal by the International Association of Space Explorers (2007). 


\section{James Biggs}

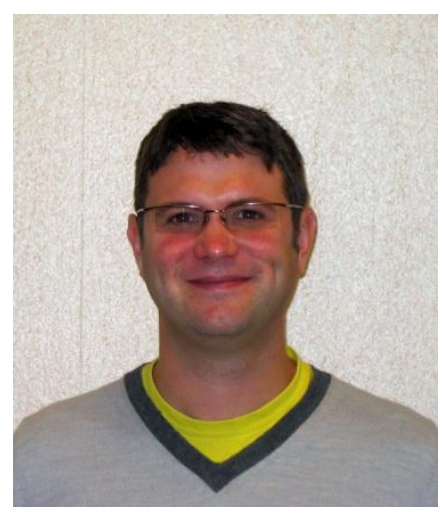

James Biggs received the B.Sc. degree in Mathematics from the University of Sussex, Brighton, UK in 1998, the M.Sc. degree in Nonlinear Dynamics and Chaos from University College London, London, U.K.

in 1999 and the PhD degree from the University of Reading, U.K. He is currently an associate director of the Advanced Space Concepts Laboratory and lecturer at the University of Strathclyde, Glasgow. Presently his research interests are focused on using dynamical systems theory, control theory and differential geometry to design novel spacecraft orbits as well as optimal attitude maneuvers.

\section{Matteo Ceriotti}

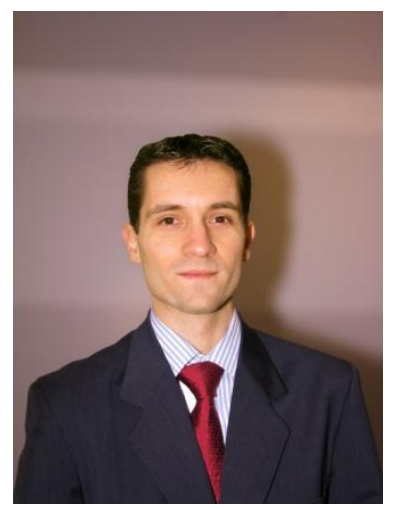

Dr. Matteo Ceriotti received his M.Sc. summa cum laude from Politecnico di Milano (Italy) in 2006 with a thesis on planning and scheduling for planetary exploration. In 2010, he received his Ph.D. on "Global Optimisation of Multiple Gravity Assist Trajectories" from the Department of Aerospace Engineering of the University of Glasgow (United Kingdom). Since 2009, Matteo is a research fellow at the Advanced Space Concepts Laboratory, University of Strathclyde, Glasgow (http://www.strath.ac.uk/space), leading the research theme "Orbital Dynamics of Large Gossamer Spacecraft". The current research topic is nonKeplerian motion of spacecraft with hybrid solar sail and solar electric propulsion. His main research interests are space mission analysis and trajectory design, orbital dynamics, trajectory optimisation, and spacecraft autonomy. He is AIAA member. 